\title{
A hierarchical sequential ALE poromechanics model for tire-soil-water interaction on fluid-infiltrated roads
}

\author{
Ines Wollny ${ }^{1}$, WaiChing Sun $^{2}$ and Michael Kaliske ${ }^{1, *, \dagger[D}$ \\ ${ }^{1}$ Institute for Structural Analysis, Technische Universität Dresden, Dresden, 01062, Germany \\ ${ }^{2}$ Department of Civil Engineering and Engineering Mechanics, Columbia University, 614 SW Mudd, 4709 New York, \\ 10027, NY, USA
}

\begin{abstract}
SUMMARY
This paper introduces a hierarchical sequential arbitrary Lagrangian-Eulerian (ALE) model for predicting the tire-soil-water interaction at finite deformations. Using the ALE framework, the interaction between a rolling pneumatic tire and the fluid-infiltrated soil underneath will be captured numerically. The road is assumed to be a fully saturated two-phase porous medium. The constitutive response of the tire and the solid skeleton of the porous medium is idealized as hyperelastic. Meanwhile, the interaction between tire, soil, and water will be simulated via a hierarchical operator-split algorithm. A salient feature of the proposed framework is the steady state rolling framework. While the finite element mesh of the soil is fixed to a reference frame and moves with the tire, the solid and fluid constituents of the soil are flowing through the mesh in the ALE model according to the rolling speed of the tire. This treatment leads to an elegant and computationally efficient formulation to investigate the tire-soil-water interaction both close to the contact and in the far field. The presented ALE model for tire-soil-water interaction provides the essential basis for future applications, for example, to a path-dependent frictional-cohesive response of the consolidating soil and unsaturated soil, respectively. Copyright (C) 2017 John Wiley \& Sons, Ltd.
\end{abstract}

Received 14 December 2016; Revised 21 February 2017; Accepted 22 February 2017

KEY WORDS: fluid-solid systems, finite element methods, tire-soil-water interaction; poromechanical ALE formulation; hierarchically staggered iteration scheme

\section{INTRODUCTION}

Vehicles are expected to spend a considerable portion of the life cycle off-road, or on pavement that is porous and permeable. Understanding the interaction between tire, water, and the solid constituent is therefore critical for predicting the durability of tires as well as of the porous subsoil. When the tire is loading the surface of the soil, the mechanical loading placed on the soil may not only trigger both deformation of the solid constituent of the soil but also lead to pore fluid seepage. This hydromechanical coupling effect can be captured via poro-elasticity theory [1,2]. Furthermore, because of the low confining pressure and the sometimes large amount of load imposed on the tire-soil contact, it is essential to incorporate geometrical nonlinearity into the hydro-mechanical simulations that capture the interactions among the tire, road, and the water infiltrating inside. Previous efforts, such as [3-7], have incorporated updated and total Lagrangian formulations to resolve this geometrical nonlinearity for consolidating porous media.

The framework for the numerical simulation of elastic bodies in rolling contact based on an arbitrary Lagrangian-Eulerian (ALE) formulation is developed by Nackenhorst [8]. ALE formulations are widely used in fluid dynamics $[9,10]$ and forming processes [11-13]. In ALE formulations, an

\footnotetext{
*Correspondence to: Michael Kaliske, Institute for Structural Analysis, Technische Universität Dresden, 01062 Dresden, Germany.

†E-mail: michael.kaliske@tu-dresden.de
} 

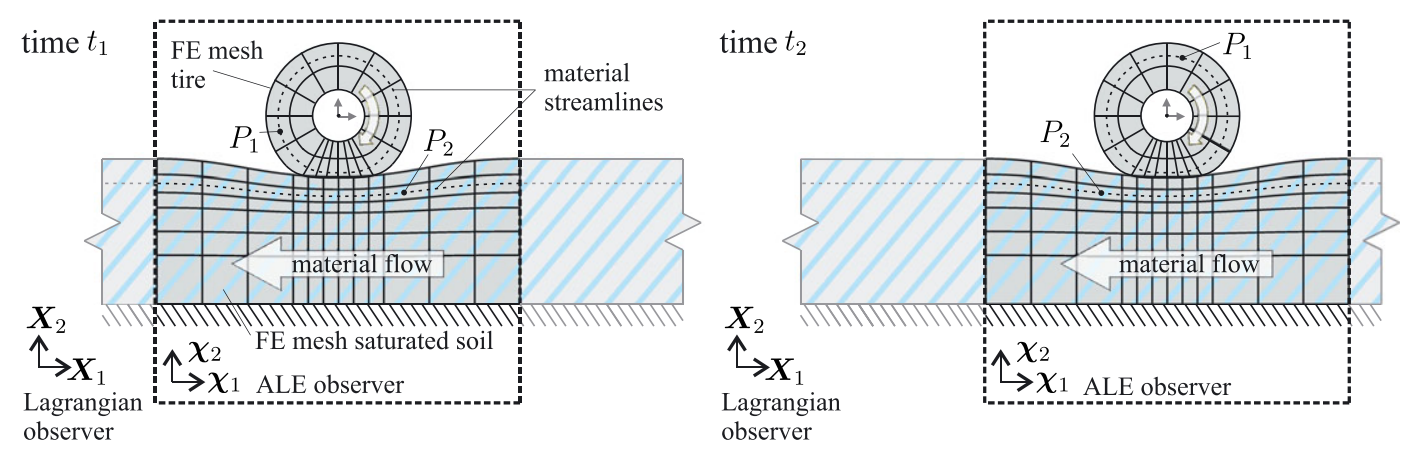

Figure 1. Lagrangian and arbitrary Lagrangian-Eulerian (ALE) observer for a steady-state rolling tire on a saturated soil.

arbitrarily moving reference frame is introduced, such that the advantages of the Lagrangian as well as of the Eulerian formulation are exploited. In case of steady state rolling tires, the reference frame moves with the tire axle through the space. The ALE formulation for steady state rolling tires is a special case of the ALE formulations, because the guiding velocity and, therefore, the movement of the arbitrary reference frame are known a priori [8]. Introducing such a reference frame for the steady state rolling tire, the deformation state of the tire, and the soil are time independent for the ALE observer, as illustrated in Figure 1. This enables a time independent formulation and compared with a Lagrangian formulation, which requires a huge number of time steps, a numerically efficient description of the dynamic process $[8,14]$. The introduction of the moving reference frame leads to a flow of the material particles (e.g., $P_{1}$ and $P_{2}$ in Figure 1) through the moving finite element mesh, which has to be considered in case of inelastic material properties, where the material history has to be collected along the material streamlines. Approaches for the consideration of inelastic tire materials in the steady state ALE frame for rolling bodies are given, for example, by the authors in [15-19] and for the pavement/subground by the authors in [14, 20]. A sequentially coupled ALE tire-pavement model, which considers inelastic tire and pavement materials, is described in [21].

On the other hand, Nazem et al. [22] has introduced an ALE poromechanics formulation to simulate cavity expansion and footing problems. In these applications, the geometrical nonlinearity is partially taken into account using the Jaumann rate formulation, but the balance of mass equation does not take into account the impact of the geometrical nonlinearity on the effective permeability and specific storage. The ALE formulation presented in [22] is neither intended nor able to capture rolling loads on the soil. Furthermore, to the best knowledge of the authors, there has yet been any effort on capturing the tire-water-soil interaction in the finite deformation range. Because the off-road mobility of a vehicle significantly depends on how soft soil deforms being subjected by vehicle loading, the capacity to capture the tire-water-soil interaction, when the soil undergoes large deformation, is critical for predicting the behavior of a vehicle on earth and on other planets [23, 24].

In the present work, our goal is to derive a new computational model that can handle tire-soil-water interaction at finite deformations. In particular, we formulate for the first time, an ALE poromechanics model for saturated soil at steady state rolling tire load. At this stage, a hyperelastic soil material behavior is assumed. Nevertheless, approaches for the consideration of inelastic materials at finite strain based on multiplicative kinematics are developed in [14] and will be included into the tiresoil-water framework in our further research. By fixing the FE mesh in the reference frame, while tracking the mass flux of the solid and fluid constituents relatively to the rolling speed of the tire, the new model provides an efficient means to simulate the tire-soil-water interaction. On the computational side, we also, for the first time, introduce a hierarchical operator-split ALE solver designed to allow the tire, solid skeleton, and pore fluid solvers advance sequentially and achieve global equilibrium through an iterative process. This split design of the algorithm allows one to divide a large and complex mixed field saddle point finite element problem into multiple sub-problems in which numerical stability can be guaranteed once the tangent of the discretized sub-problem is positive 
definite. The drained and undrained split ALE strategy are compared through numerical experiments to demonstrate the robustness and accuracy of the proposed model.

The rest of this paper is organized as follows. In Section 2, we first describe the ALE formulation for the poromechanics problem. The finite element implementation of the iterative sequential fluidsolid coupling solver is described in Section 3. The implementation of the ALE solid-fluid solver into the staggered tire-pavement iteration scheme is shown in Section 4, where it is also applied to an engineering problem that predicts the interaction between a truck tire and a saturated hyperelastic soil. The presented research is summarized in Section 5. The Appendix provides further the FE equations of the mass balance in Lagrangian formulation and a verification of the transient Lagrangian solid-fluid solver on the basis of the analytical solution of Terzaghi's problem. This verification example provides also a stability analysis of the different iterative solver types. Based on the transient Lagrangian solid-fluid computations, the Appendix includes additionally a verification of the poromechanical ALE solid-fluid formulation.

As for notations and symbols, bold-faced letters denote first-order tensors, bold-faced and underlined letters stand for second-order tensors, and double underlined letters for fourth-order tensors. The symbol ' ' denotes a single contraction of adjacent indices of two tensors (e.g., $\boldsymbol{a} \cdot \boldsymbol{b}=a_{i} b_{i}$ or $\underline{\boldsymbol{c}} \cdot \underline{\boldsymbol{d}}=c_{i j} d_{j k}$ ), the symbol ' $:$ ' indicates a double contraction of adjacent indices of tensors of rank two or higher ( e.g., $\underline{\underline{\boldsymbol{C}}}: \underline{\boldsymbol{\epsilon}}^{\boldsymbol{e}}=C_{i j k l} \epsilon_{k l}^{e}$ ) and the symbol ' $\otimes$ ' denotes a dyadic product of two vectors (e.g. $\left.\boldsymbol{a} \otimes \boldsymbol{b}=a_{i} b_{j}\right)$ or two second-order tensors (e.g. $\left.(\underline{\boldsymbol{\alpha}} \otimes \boldsymbol{\beta})=\alpha_{i j} \beta_{k l}\right)$. As for sign convention, we consider the direction of the solid tensile stress and the fluid pore pressure as positive.

\section{POROMECHANICAL ARBITRARY LAGRANGIAN-EULERIAN FORMULATION}

\subsection{Arbitrary Lagrangian-Eulerian kinematics}

Unlike single-phase continua or composites undergoing compatible deformation, the fluid and solid constituents of a fully saturated porous medium may each move at different trajectories. Therefore, a controlled mass of solid constituents may contain fluid in the deformed void space that was not necessarily present in the void space of the solid skeleton in the reference configuration. In what follows, we derive the balance principles based on the solid trajectory and the solid deformation mapping. As a result, all material time derivatives and spatial derivatives are expressed in terms of the solid motion. For brevity, the designation of the solid phase $s$ is dropped.

In the current configuration, the solid and the fluid constituents occupy the combined space $\Phi(\mathcal{B})$, while in the initial configuration, $\mathcal{B}$ contains the solid phase and $\mathcal{B}^{f}$ contains the pore fluid. The mapping from the initial to the current configuration is achieved by $\Phi(X, t)$ for the solid and by $\Phi^{f}\left(\boldsymbol{X}^{f}, t\right)$ for the fluid. By introducing an additional reference configuration in the ALE kinematics, where the solid phase is in space $\chi(\mathcal{B})$ and the fluid phase is described by $\chi\left(\mathcal{B}^{f}\right)$, the mapping from the initial to the current configuration can be split into the rigid body motion $\chi(\mathcal{B})$ and $\chi\left(\mathcal{B}^{f}\right)$ from the initial to the reference configuration and into the deformation $\hat{\Phi}(\chi, t)$ and $\hat{\Phi}^{f}\left(\chi^{f}, t\right)$ of the solid and the fluid, respectively, from the reference to the current configuration, see Figure 2 . Therefore, the current position $\boldsymbol{x}$ of a point $P$ at time $t$ of combined solid and fluid can be expressed in terms of the initial solid position $\boldsymbol{X}$ by

$$
\boldsymbol{x}=\Phi(X, t)=\hat{\Phi}(\chi(X, t))
$$

and in terms of the initial fluid position $\boldsymbol{X}^{f}$ by

$$
\boldsymbol{x}=\Phi^{f}\left(X^{f}, t\right)=\hat{\Phi}^{f}\left(\chi\left(X^{f}, t\right)\right) .
$$

Further, a moving reference coordinate system $e_{i}^{\mathrm{ALE}}$ is introduced in the ALE frame. Then, the current position of a point $P$ might be expressed in several ways by

$$
\boldsymbol{x}=\boldsymbol{X}+\boldsymbol{u}=\chi_{\boldsymbol{0}}^{\mathrm{ALE}}+\boldsymbol{\varphi}=\chi_{\boldsymbol{0}}^{\mathrm{ALE}}+\chi+\hat{\boldsymbol{u}}=\boldsymbol{X}+\boldsymbol{u}_{\mathrm{rig}}+\hat{\boldsymbol{u}} .
$$




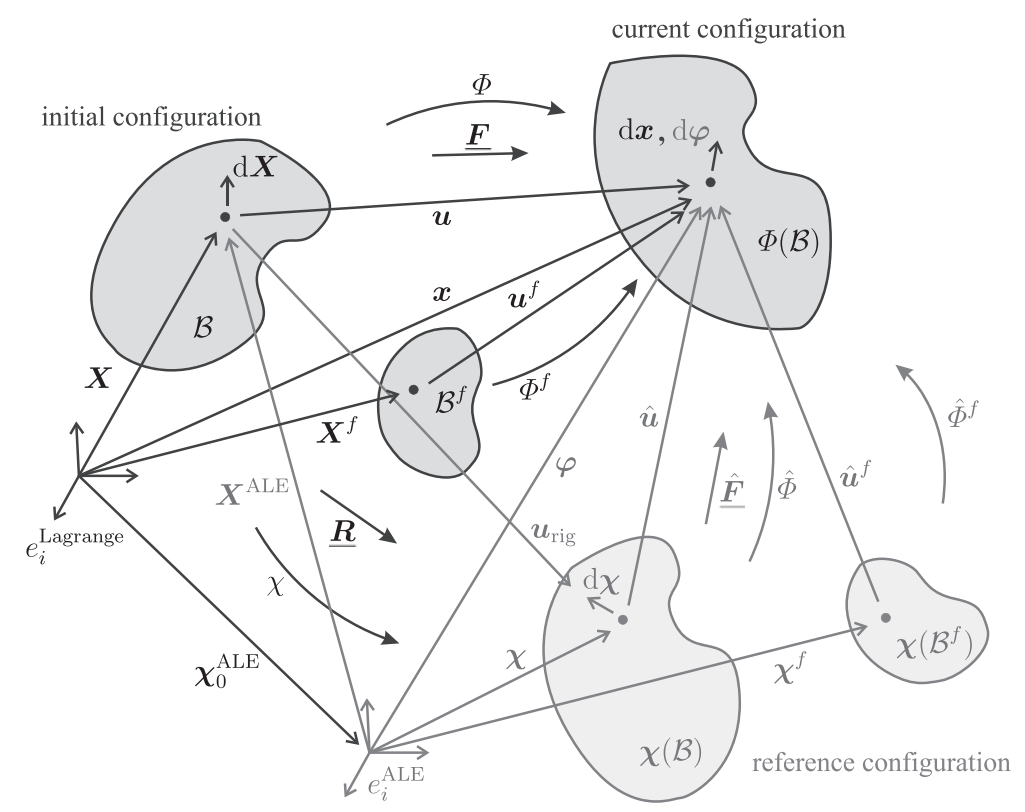

Figure 2. Poromechanical arbitrary Lagrangian-Eulerian (ALE) kinematics.

The material time derivative of a scalar value $f[25]$ in the reference frame is given by

$$
\dot{f}=\left.\frac{\partial f}{\partial t}\right|_{X}=\left.\frac{\partial f}{\partial t}\right|_{\chi}+\nabla^{\chi} f \cdot \boldsymbol{w}
$$

with

$$
\nabla^{\chi} f=\operatorname{Grad} f=\frac{\partial f}{\partial \chi}
$$

and

$$
w=\left.\frac{\partial \chi}{\partial t}\right|_{X}
$$

where $\boldsymbol{w}$ is called guiding velocity and obtained by Equations (3) and (4) as

$$
\boldsymbol{w}=\left.\frac{\partial \chi}{\partial t}\right|_{X}=\left.\frac{\partial\left(\boldsymbol{X}+\boldsymbol{u}_{\mathrm{rig}}-\chi_{\mathbf{0}}^{\mathrm{ALE}}\right)}{\partial t}\right|_{X}=\left.\frac{\partial \boldsymbol{u}_{\mathrm{rig}}}{\partial t}\right|_{X}-\left.\frac{\partial \chi_{\mathbf{0}}^{\mathrm{ALE}}}{\partial t}\right|_{X} .
$$

In case of a pavement or a soil, which performs no rigid body motion $\boldsymbol{u}_{\mathrm{rig}}=0$, the guiding velocity yields

$$
\boldsymbol{w}_{\mathrm{pav}}=\left.\frac{\partial \chi}{\partial t}\right|_{X}=-\left.\frac{\partial \chi_{0}^{\mathrm{ALE}}}{\partial t}\right|_{X}
$$

and, therefore, equals the negative value of the velocity of the moving reference coordinate system.

The material velocity $\boldsymbol{v}(\boldsymbol{x}, t)$ of a solid particle in the current configuration with respect to the fixed initial frame follows from the material time derivative of Equation (4) as

$$
v(x, t)=\dot{x}=\left.\frac{\partial x}{\partial t}\right|_{X}=\left.\frac{\partial x}{\partial t}\right|_{\chi}+\nabla^{\chi} x \cdot w
$$


With $\boldsymbol{x}=\chi_{\mathbf{0}}^{\mathrm{ALE}}+\boldsymbol{\varphi}$ and $\nabla^{\chi} \chi_{\mathbf{0}}^{\mathrm{ALE}}=0$, Equation (9) reads

$$
\boldsymbol{v}(\boldsymbol{x}, t)=\left.\frac{\partial \chi_{\mathbf{0}}^{\mathrm{ALE}}}{\partial t}\right|_{\chi}+\left.\frac{\partial \boldsymbol{\varphi}}{\partial t}\right|_{\chi}+\nabla^{\chi} \boldsymbol{\varphi} \cdot \boldsymbol{w}=\left.\frac{\partial \chi_{\mathbf{0}}^{\mathrm{ALE}}}{\partial t}\right|_{\chi}+\dot{\boldsymbol{\varphi}}=\boldsymbol{v}^{\mathrm{ALE}}(t)+\boldsymbol{v}(\boldsymbol{\varphi}, t),
$$

where $\boldsymbol{v}^{\mathrm{ALE}}(t)$ equals the velocity of the moving reference coordinate frame and $\boldsymbol{v}(\boldsymbol{\varphi}, t)$ is the material velocity of a particle in the current configuration with respect to the moving reference frame.

The material velocity of a fluid particle is derived analogously and is given by

$$
\boldsymbol{v}^{f}(\boldsymbol{x}, t)=\left.\frac{\partial \chi_{\mathbf{0}}^{\mathrm{ALE}}}{\partial t}\right|_{\chi}+\left.\frac{\partial \boldsymbol{\varphi}}{\partial t}\right|_{\chi^{f}}+\nabla^{\chi^{f}} \boldsymbol{\varphi} \cdot \boldsymbol{w}^{f}=\boldsymbol{v}^{\mathrm{ALE}}(t)+\boldsymbol{v}^{f}(\boldsymbol{\varphi}, t),
$$

where $\boldsymbol{v}^{f}(\boldsymbol{\varphi}, t)$ is the material velocity of a fluid particle in the current configuration with respect to the moving reference frame and $\nabla \chi^{f} \boldsymbol{\varphi}=\operatorname{Grad}^{f} \boldsymbol{\varphi}=\partial \varphi / \partial \chi^{f}$. The relative velocity $\tilde{\boldsymbol{v}}$ between solid and fluid phase is

$$
\tilde{v}(x, t)=v^{f}(x, t)-v(x, t)=v^{f}(\varphi, t)-v(\varphi, t)=\tilde{v}(\varphi, t)
$$

and, therefore, the same with respect to the fixed coordinate system $e_{i}^{\text {Lagrange }}$ and to the moving coordinate system $e_{i}^{\mathrm{ALE}}$.

Using $x=\chi_{0}^{\mathrm{ALE}}+\boldsymbol{i}$ and $\partial \chi_{0}^{\mathrm{ALE}} / \partial X=0$, the deformation gradient can be expressed as

$$
\underline{\boldsymbol{F}}=\frac{\partial \boldsymbol{x}}{\partial X}=\nabla^{X} \boldsymbol{x}=\operatorname{GRAD} \boldsymbol{x}=\frac{\partial \varphi}{\partial X}=\frac{\partial \varphi}{\partial \chi} \cdot \frac{\partial \chi}{\partial X}=\underline{\hat{\boldsymbol{F}}} \cdot \underline{\boldsymbol{R}},
$$

where $\hat{\boldsymbol{F}}$ is the deformation gradient with respect to the reference configuration and $\underline{\boldsymbol{R}}$ contains the rigid body rotations from the initial to the reference configuration with $\operatorname{det} \underline{R}=1$. The Jacobian is then obtained by

$$
J=\operatorname{det} \underline{\boldsymbol{F}}=\operatorname{det} \underline{\hat{\boldsymbol{F}}}=\hat{J}>0 .
$$

By homogenization of the solid-fluid mixture as continuum, the density of a fully saturated porous medium is given $[5,6,26-28]$ as

$$
\rho=\rho^{s}+\rho^{f}=\phi^{s} \rho_{s}+\phi^{f} \rho_{f} .
$$

Thereby, $\rho_{\alpha}$ with $\alpha=s, f$ is defined as the mass of the constituent $\alpha$ divided by the current volume of the constituent $\alpha$, while $\rho^{\alpha}$ is the partial density of the $\alpha$ constituent, obtained by the mass of the constituent $\alpha$ divided by the volume of the mixture in the current configuration. The volume fraction of the solid constituent in the current configuration is $\phi^{s}$, and $\phi^{f}$ is the porosity of the porous medium in the current configuration, which is referred as Eulerian porosity in [29]. The current porosity can be obtained from the porosity $\hat{\phi}^{\mathrm{f}}$ in the reference frame by $\phi^{f}=\hat{\phi}^{f} / J$. For fully saturated porous media, $\phi^{s}+\phi^{f}=1$ holds. Thus, the total current density can be written as

$$
\rho=\left(1-\phi^{f}\right) \rho_{s}+\phi^{f} \rho_{f},
$$

whereby the densities of both constituents depend on the pore pressure.

In addition to the standard continuum mechanical stress measures of the total Cauchy stress tensor $\underline{\boldsymbol{\sigma}}$, the first total Piola-Kirchhoff stress tensor $\underline{\boldsymbol{P}}=J \underline{\boldsymbol{\sigma}} \cdot \underline{\boldsymbol{F}}^{-T}$ and the second total Piola-Kirchhoff stress tensor $\underline{\boldsymbol{S}}=\underline{\boldsymbol{F}}^{-1} \cdot \underline{\boldsymbol{P}}$ (compare, e.g., [30]), the first and second total Piola-Kirchhoff stress tensors are further defined with respect to the reference frame as

$$
\underline{\hat{\boldsymbol{P}}}=J \underline{\boldsymbol{\sigma}} \cdot \underline{\hat{\boldsymbol{F}}}^{-T} \text { and } \quad \underline{\hat{\boldsymbol{S}}}=\underline{\hat{\boldsymbol{F}}}^{-1} \cdot \underline{\hat{\boldsymbol{P}}}
$$


respectively. These total stress measures can be partitioned additively, according to the effective stress principle $[1,2]$, into two parts: the contributions of the solid skeleton and of the fluid constituents. As a result, the total Cauchy stress of a porous medium is the sum of solid and fluid,

$$
\underline{\sigma}=\underline{\sigma}^{s}+\underline{\sigma}^{f}=\phi^{s} \underline{\boldsymbol{\sigma}}_{s}+\phi^{f} \underline{\boldsymbol{\sigma}}_{f} .
$$

The intrinsic partial Cauchy stresses $\underline{\boldsymbol{\sigma}}_{s}$ and $\underline{\boldsymbol{\sigma}}_{f}$ are defined in the volume of the solid grains $V^{s}$ and pore space $V^{f}$, respectively. The volume averaged stress defined in the current volume $V=V^{s}+V^{f}$ is the total Cauchy stress. When the shear resistance of the pore fluid is neglected, the intrinsic partial stress of the fluid constituent $\underline{\sigma}_{f}$ is isotropic and depends on the intrinsic pore pressure $p_{f}$. The partial total stress of the fluid constituent $\underline{\sigma}^{f}$ is subsequently obtained in terms of the macroscopic pore pressure $p^{f}$ by

$$
\underline{\boldsymbol{\sigma}}^{f}=\phi^{f} \underline{\boldsymbol{\sigma}}_{f}=-\phi^{f} p_{f} \underline{\boldsymbol{I}}=-p^{f} \underline{\boldsymbol{I}} .
$$

The partial total stress of the solid constituent $\sigma^{s}$ depends on the effective stress $\underline{\sigma}^{\prime}$ and the stress applied to the solid grains by the pore fluid $K p^{f} / K_{s} \underline{\boldsymbol{I}}$, that is,

$$
\underline{\boldsymbol{\sigma}}^{s}=\underline{\boldsymbol{\sigma}}^{\prime}+\frac{K}{K_{s}} p^{f} \underline{\boldsymbol{I}}
$$

Substituting (19) and (20) into (18), the total Cauchy stress reads subsequently

$$
\underline{\boldsymbol{\sigma}}=\underline{\boldsymbol{\sigma}}^{\prime}-B p^{f} \underline{\boldsymbol{I}}
$$

where $B$ is Biot's coefficient defined as

$$
B=1-\frac{K}{K_{s}}
$$

with the bulk modulus $K_{\alpha}$ of the constituent $\alpha$ and the bulk modulus of the solid grain skeleton $K$. Typically, Biot's coefficient $B$ is close to unity for sand. Using Piola transformation, the first total Piola-Kirchhoff stress tensor is given by

$$
\underline{\boldsymbol{P}}\left(\underline{\boldsymbol{F}}, p^{f}\right)=\underline{\boldsymbol{P}}^{\prime}(\underline{\boldsymbol{F}})-J B p^{f} \underline{\boldsymbol{F}}^{-T} \quad \text { with } \quad \underline{\boldsymbol{P}}^{\prime}=J \underline{\boldsymbol{\sigma}}^{\prime} \cdot \underline{\boldsymbol{F}}^{-T}
$$

with respect to the initial configuration and by

$$
\underline{\hat{\boldsymbol{P}}}\left(\underline{\hat{\boldsymbol{F}}}, p^{f}\right)=\underline{\hat{\boldsymbol{P}}}^{\prime}(\underline{\hat{\boldsymbol{F}}})-J B p^{f} \underline{\hat{\boldsymbol{F}}}^{-T} \text { with } \quad \underline{\hat{\boldsymbol{P}}}^{\prime}=J \underline{\boldsymbol{\sigma}}^{\prime} \cdot \underline{\hat{\boldsymbol{F}}}^{-T}
$$

with respect to the reference frame. The second total Piola-Kirchhoff stress tensor with respect to the reference frame, which is used for the later implementation, reads

$$
\underline{\hat{\boldsymbol{S}}}\left(\underline{\hat{\boldsymbol{F}}}, p^{f}\right)=\underline{\hat{\boldsymbol{F}}}^{-1} \cdot \underline{\hat{\boldsymbol{P}}}=\underline{\hat{\boldsymbol{F}}}^{-1} \cdot \underline{\hat{\boldsymbol{P}}}^{\prime}-J B p^{f} \underline{\hat{\boldsymbol{F}}}^{-1} \cdot \underline{\hat{\boldsymbol{F}}}^{-T} .
$$

\subsection{Balance of linear momentum}

The strong form of the balance of momentum with respect to the current configuration (compare, e.g., [30]) is given by

$$
\rho \dot{\boldsymbol{v}}=\nabla^{\boldsymbol{x}} \cdot \underline{\boldsymbol{\sigma}}+\rho \boldsymbol{G}+\boldsymbol{h}^{s}+\boldsymbol{h}^{f}
$$

and with respect to the reference configuration (compare [8]), it reads

$$
\hat{\rho} \dot{\boldsymbol{v}}=\nabla^{\chi} \cdot \underline{\hat{\boldsymbol{P}}}+\hat{\rho} \boldsymbol{G}+J \boldsymbol{h}^{s}+J \boldsymbol{h}^{f} .
$$

Thereby, $\rho$ is the density in the current and $\hat{\rho}$ in the reference frame. Gravity is represented by $\boldsymbol{G}$ and the divergence is $\nabla^{x} \cdot \underline{\boldsymbol{A}}=\operatorname{div} \underline{\boldsymbol{A}}=\partial \underline{\boldsymbol{A}}_{a b} / \partial \boldsymbol{x}_{b}$ in the current and $\nabla^{\chi} \cdot \underline{\boldsymbol{A}}=\operatorname{Div} \underline{\boldsymbol{A}}=\partial \underline{\boldsymbol{A}}_{a b} / \partial \chi_{b}$ in the 
reference configuration. $\boldsymbol{h}^{s}$ and $\boldsymbol{h}^{f}$ are the interactive body forces per unit reference volume exerted on their corresponding phases due to drag, lift, virtuall mass effect, history effects, and the relative spinning (Magnus effect), which balance out internally, that is, $\boldsymbol{h}^{s}+\boldsymbol{h}^{f}=\mathbf{0}$. In both equations, $\dot{\boldsymbol{v}}$ is the time derivative of the material velocity $\boldsymbol{v}(\boldsymbol{x}, t)$, which is obtained by

$$
\begin{aligned}
\dot{\boldsymbol{v}}(\boldsymbol{x}, t) & =\left.\frac{\partial \boldsymbol{v}(\boldsymbol{x}, t)}{\partial t}\right|_{\chi}+\nabla^{\chi} \boldsymbol{v}(\boldsymbol{x}, t) \cdot \boldsymbol{w} \\
& =\left.\frac{\partial \boldsymbol{v}^{\mathrm{ALE}}(t)}{\partial t}\right|_{\chi}+\left.\frac{\partial \boldsymbol{v}(\boldsymbol{\varphi}, t)}{\partial t}\right|_{\chi}+\nabla^{\chi}\left(\boldsymbol{v}^{\mathrm{ALE}}(t)+\boldsymbol{v}(\boldsymbol{\varphi}, t)\right) \cdot \boldsymbol{w} .
\end{aligned}
$$

Because $v^{\mathrm{ALE}}(t)$ is independent of the position $\chi$, it holds that $\nabla \chi v^{\mathrm{ALE}}=0$. In case of a steady state rolling or moving (constant rotational and translational velocities), further, the time derivatives with respect to the reference frame vanish, and it follows $\dot{\boldsymbol{v}}(\boldsymbol{x}, t)=\dot{\boldsymbol{v}}(\varphi, t)$.

\subsection{Balance of mass}

The balance of mass for the pore fluid constituent in the current configuration of the solid skeleton reads $[6,7,31]$,

$$
\dot{\rho}^{f}+J \nabla^{x} \cdot \omega=0
$$

whereby $\boldsymbol{\omega}$ is the Eulerian relative mass flow defined as

$$
\omega=\Phi^{f} \rho_{f}\left(v^{f}-v\right)
$$

The relative mass flow with respect to the reference configuration reads

$$
\hat{\boldsymbol{\omega}}=J \underline{\hat{\boldsymbol{F}}}^{-1} \cdot \omega .
$$

Then, the balance of mass with respect to the reference configuration is obtained as

$$
\dot{\rho}^{f}+\nabla^{\chi} \cdot \hat{\boldsymbol{\omega}}=0
$$

Assuming isothermal conditions, the material time derivative of the pore fluid density can be partitioned as

$$
\dot{\rho}^{f}=\Phi^{f} \dot{\rho}_{f}+\rho_{f} \dot{\Phi}^{f}
$$

Further, assuming an incompressible fluid with $\dot{\rho}_{f}=0$, Equation (33) simplifies to

$$
\dot{\rho}^{f}=\rho_{f} \dot{\Phi} f .
$$

For two-phase porous media composed of incompressible solid constituents, Biot's coefficient becomes $B=1$, and Biot's modulus $M$ tends to infinity. Then, the material time derivative of the pore fluid density can be expressed as $\dot{\rho}^{f}=\rho_{f} \dot{J} / J$, and the mass balance reads

$$
\rho_{f} \frac{\dot{J}}{J}+\nabla^{\chi} \cdot \hat{\boldsymbol{\omega}}=0
$$

In [6], the time derivative of the apparent fluid density $\rho^{f}$ in case of compressible solids and fluids is 
derived as

$$
\dot{\rho}^{f}=\rho_{f}\left[\dot{B}\left(\log J+\frac{p^{f}}{K_{s}}\right)+\frac{B}{J} \dot{J}+\frac{1}{M} \dot{p}^{f}\right] .
$$

Assuming $\dot{B} \approx 0$, the mass balance is given by

$$
\rho_{f} B \frac{\dot{J}}{J}+\frac{\rho_{f}}{M} \dot{p}^{f}+\nabla^{\chi} \cdot \hat{\boldsymbol{\omega}}=0,
$$

where Biot's modulus is a function of the current porosity, the bulk moduli of the solid skeleton, and of the solid and fluid constituents,

$$
M=\frac{K_{s} K_{f}}{K_{f}\left(B-\phi^{f}\right)+K_{s} \phi^{f}} .
$$

Assuming that the pore fluid flow is Darcian, the relative pore fluid mass flux is given by

$$
\boldsymbol{\omega}=\rho_{f} \underline{\boldsymbol{k}} \cdot\left[-\nabla^{x} p^{f}+\rho_{f}\left(\boldsymbol{G}-\boldsymbol{a}^{f}\right)\right],
$$

where $\boldsymbol{k}$ is the permeability tensor divided by the viscosity and $\boldsymbol{a}^{f}$ is the acceleration of the fluid constituent. By multiplying Equation (39) with $J \underline{\hat{\boldsymbol{F}}}^{-1}$ from the left

$$
\hat{\boldsymbol{\omega}}=\rho_{f} J \underline{\hat{\boldsymbol{F}}}^{-1} \cdot \underline{\boldsymbol{k}} \cdot \underline{\hat{\boldsymbol{F}}}^{-T} \cdot \underline{\hat{\boldsymbol{F}}}^{T}\left[-\nabla^{\boldsymbol{x}} p^{f}+\rho_{f}\left(\boldsymbol{G}-\boldsymbol{a}^{f}\right)\right],
$$

by introducing $\underline{\hat{\boldsymbol{K}}}=J \underline{\hat{\boldsymbol{F}}}^{-1} \cdot \underline{\boldsymbol{k}} \cdot \underline{\hat{\boldsymbol{F}}}^{-T}$ and by transformation $-\underline{\hat{\boldsymbol{F}}}^{T} \nabla^{\boldsymbol{x}} p^{f}=-\nabla x p^{f}$, the relative pore fluid mass flux with respect to the reference configuration is obtained as

$$
\hat{\boldsymbol{\omega}}=\rho_{f} \underline{\hat{\boldsymbol{K}}} \cdot\left[-\nabla^{\chi} p^{f}+\rho_{f} \hat{\boldsymbol{F}}^{T} \cdot\left(\boldsymbol{G}-\boldsymbol{a}^{f}\right)\right] .
$$

Inserting Equation (41) into Equation (35) and assuming the simplifications, that the gravity as well as the inertia force of the fluid are negligible $\boldsymbol{G}=\boldsymbol{a}^{f}=\mathbf{0}$, modifies the balance of mass in case of incompressible solid and fluid constituents to

$$
\frac{\dot{J}}{J}-\nabla^{\chi} \cdot\left(\underline{\hat{\boldsymbol{K}}} \cdot \nabla^{\chi} p^{f}\right)=0
$$

and in case of compressible solids and fluids to

$$
B \frac{\dot{J}}{J}+\frac{1}{M} \dot{p}^{f}-\nabla^{\chi} \cdot\left(\underline{\hat{\boldsymbol{K}}} \cdot \nabla^{\chi} p^{f}\right)=0
$$

\section{FINITE ELEMENT IMPLEMENTATION}

\subsection{Weak form and linearization of balance of momentum}

The balance of momentum is given in its weak form by

$$
\int_{\chi(B)} \hat{\rho} \dot{\boldsymbol{v}} \cdot \boldsymbol{\eta} \mathrm{d} \hat{V}+\int_{\chi(B)} \underline{\hat{\boldsymbol{P}}}: \operatorname{Grad} \boldsymbol{\eta} \mathrm{d} \hat{V}=\int_{\chi(B)} \hat{\rho} \boldsymbol{G} \cdot \boldsymbol{\eta} \mathrm{d} \hat{V}+\int_{\partial_{t} \chi(B)} \hat{\boldsymbol{T}} \cdot \boldsymbol{\eta} \mathrm{d} \hat{\mathrm{A}}
$$

with $\underline{\hat{\boldsymbol{P}}} \cdot \hat{\boldsymbol{N}}=\hat{\boldsymbol{T}}$. For the a staggered fluid-solid iteration scheme, it is linearized with respect to $\boldsymbol{\varphi}$ with $\boldsymbol{\varphi}_{(i+1)}^{(k)}=\boldsymbol{\varphi}_{(i)}^{(k)}+\Delta \boldsymbol{\varphi}$, where $i$ indicates the solid iteration step and $k$ is the iteration step of the staggered fluid-solid iteration. The linearization of the balance of momentum is carried out for different assumptions concerning the pore pressure and the mass balance equation, respectively, during the evaluation of the balance of momentum (solid step). 
3.1.1. Drained split. In a drained split, it is assumed that the fluid mass content might change (drainage) during the solid step, while the pore pressure is constant. Because the ALE formulation is related to the reference frame, it is assumed that $\underline{\hat{S}}^{f}=-J B p^{f} \underline{\boldsymbol{F}}^{-1} \cdot \underline{\hat{\boldsymbol{F}}}^{-T}$ is fixed during the solid step. In this case, the linearization of the internal forces in the reference frame by $\underline{\boldsymbol{P}}_{(i+1)}=\underline{\hat{\boldsymbol{P}}}_{(i)}+\Delta \underline{\hat{\boldsymbol{P}}}$ is similar to a Lagrangian linearization and leads by replacing $\underline{\hat{\boldsymbol{P}}}=\underline{\hat{\boldsymbol{F}}} \cdot \underline{\hat{\boldsymbol{S}}}=\underline{\hat{\boldsymbol{F}}} \cdot \underline{\hat{\boldsymbol{\prime}}}+\underline{\hat{\boldsymbol{F}}} \cdot \underline{\hat{\boldsymbol{S}}}^{f}$ to

$$
\begin{aligned}
& \int_{\chi(B)} \hat{\boldsymbol{P}}_{(i+1)}: \operatorname{Grad} \boldsymbol{\eta} \mathrm{d} \hat{V}=\int_{\chi(B)}\left(\underline{\hat{\boldsymbol{F}}} \cdot{\underline{\boldsymbol{S}^{\prime}}}_{{ }_{(i+1)}}: \operatorname{Grad} \boldsymbol{\eta} \mathrm{d} \hat{V}\right. \\
& +\int_{\chi(B)}\left(\underline{\hat{\boldsymbol{F}}} \cdot \underline{\hat{S}}^{f}\right)_{(i+1)}: \operatorname{Grad} \boldsymbol{\eta} \mathrm{d} \hat{V} \\
& =\int_{\chi(B)}\left(\underline{\hat{\boldsymbol{F}}} \cdot \underline{\hat{\boldsymbol{S}}}^{\prime}\right)_{(i)}: \operatorname{Grad} \boldsymbol{\eta} \mathrm{d} \hat{V} \\
& +\int_{\chi(B)}\left(\Delta \underline{\hat{\boldsymbol{F}}} \cdot{\underline{\hat{\boldsymbol{S}}^{\prime}}}_{(i)}+\underline{\hat{\boldsymbol{F}}}_{(i)} \cdot \Delta{\underline{\boldsymbol{S}^{\prime}}}^{\prime}: \operatorname{Grad} \boldsymbol{\eta} \mathrm{d} \hat{V}\right. \\
& +\int_{\chi(B)}\left(\underline{\hat{\boldsymbol{F}}} \cdot \underline{\hat{S}}^{f}\right)_{(i)}: \operatorname{Grad} \boldsymbol{\eta} \mathrm{d} \hat{V} \\
& +\int_{\chi(B)}\left(\Delta \underline{\hat{\boldsymbol{F}}} \cdot \underline{\hat{\boldsymbol{S}}}^{f}\right): \operatorname{Grad} \boldsymbol{\eta} \mathrm{d} \hat{V} \\
& =\int_{\chi(B)}\left(\underline{\hat{\boldsymbol{F}}}_{(i)} \cdot \underline{\hat{\boldsymbol{S}}}_{(i)}\right): \operatorname{Grad} \boldsymbol{\eta} \mathrm{d} \hat{V} \\
& +\int_{\chi(B)}\left(\operatorname{Grad} \Delta \boldsymbol{\varphi} \cdot \underline{\hat{\boldsymbol{S}}}_{(i)}+\underline{\hat{\boldsymbol{F}}}_{(i)} \cdot \Delta \underline{\hat{\boldsymbol{S}}}^{\prime}\right): \operatorname{Grad} \boldsymbol{\eta} \mathrm{d} \hat{V},
\end{aligned}
$$

whereby the increment of the effective second Piola-Kirchhoff stress tensor is derived from the constitutive relation

$$
\begin{aligned}
& \Delta \underline{\hat{\boldsymbol{S}^{\prime}}}=\left.\frac{\partial \underline{\hat{\boldsymbol{S}}^{\prime}}}{\partial \underline{\hat{\boldsymbol{C}}}}\right|_{\boldsymbol{\varphi}_{(i)}}: \Delta \underline{\hat{\boldsymbol{C}}}=\left.2 \frac{\partial \underline{\hat{\boldsymbol{S}^{\prime}}}}{\partial \underline{\hat{\boldsymbol{C}}}}\right|_{\boldsymbol{\varphi}_{(i)}}: \Delta \underline{\hat{\boldsymbol{E}}} \\
& =\underline{\underline{\mathbb{C}}}_{(i)}: \Delta \underline{\hat{\boldsymbol{E}}}=\underline{\underline{\hat{\mathbb{C}}}}_{(i)}: \frac{1}{2}\left[\underline{\hat{\boldsymbol{F}}}_{(i)}^{T} \cdot \operatorname{Grad} \Delta \varphi+\operatorname{Grad}^{T} \Delta \varphi \cdot \underline{\hat{\boldsymbol{F}}}_{(i)}\right] \text {. }
\end{aligned}
$$

3.1.2. Undrained split. In case of a so-called undrained split, the balance of momentum is linearized assuming that the mass content does not change during the solid step. This is achieved by prescribing $\nabla x \cdot \hat{\boldsymbol{\omega}}=0$ in Equation (37), which yields

$$
B \frac{\dot{J}}{J}+\frac{1}{M} \dot{p}^{f}=0
$$

From the of mass balance equation, introducing $\dot{J} / J=(\ln J)[6]$,

$$
\frac{p_{n+1}^{f}-p_{n}^{f}}{\Delta t}=-M B \frac{\ln J_{n+1}-\ln J_{n}}{\Delta t}
$$

is obtained, where $n$ indicates the fixed starting point of the solid step with $p_{n}^{f}=p^{f(k-1)}$ and 
$J_{n}=J\left(\varphi^{(k-1)}\right)=J^{(k-1)}$, while $n+1$ indicates the updated state at $\varphi^{(k)}$. A prediction of the updated pressure $p^{\star(k)}=p_{n+1}^{f}$ is then obtained by rearrangement of Equation (48) as

$$
p^{\star(k)}=-M B\left(\ln J^{(k)}-\ln J^{(k-1)}\right)+p^{f(k-1)} .
$$

For the linearization of the balance of momentum with respect to $\varphi$, the first Piola-Kirchhoff stress is then replaced by $\underline{\hat{\boldsymbol{P}}}=\underline{\hat{\boldsymbol{F}}} \cdot \underline{\hat{\boldsymbol{S}}}^{\prime}+\underline{\hat{\boldsymbol{P}}}^{f \star}$ with

$$
\underline{\hat{\boldsymbol{P}}}^{f \star}=J^{(k)} B^{2} M\left(\ln J^{(k)}-\ln J^{(k-1)}\right) \underline{\hat{\boldsymbol{F}}}^{(k)^{-T}}-J^{(k)} B p^{f(k-1)} \underline{\hat{\boldsymbol{F}}}^{(k)^{-T}} .
$$

The linearization of Equation (50) with respect to $\varphi$ at fixed $J^{(k-1)}$ and $p^{f(k-1)}$ and assuming $\partial M / \partial \varphi$ $\approx 0$ yields

$$
\begin{aligned}
\underline{\hat{\boldsymbol{P}}}_{(i+1)}^{f \star}= & \underline{\hat{\boldsymbol{P}}}_{(i)}^{f \star}+\frac{\partial \underline{\hat{\boldsymbol{P}}}^{f \star}}{\partial \boldsymbol{\varphi}} \cdot \Delta \boldsymbol{\varphi} \\
= & J_{(i)} B^{2} M\left(\ln J_{(i)}-\ln J^{(k-1)}\right) \underline{\hat{\boldsymbol{F}}}_{(i)}^{-T}-J_{(i)} B p^{f(k-1)} \underline{\hat{\boldsymbol{F}}}_{(i)}^{-T} \\
& +\frac{\partial J}{\partial \boldsymbol{\varphi}} \cdot \Delta \boldsymbol{\varphi} B^{2} M\left(\ln J_{(i)}-\ln J^{(k-1)}\right) \hat{\hat{\boldsymbol{F}}}_{(i)}^{-T} \\
& +J_{(i)} B^{2} M \frac{\partial \ln J}{\partial \varphi} \cdot \Delta \varphi \underline{\hat{\boldsymbol{F}}}_{(i)}^{-T}+J_{(i)} B^{2} M\left(\ln J_{(i)}-\ln J^{(k-1)}\right) \frac{\partial \underline{\boldsymbol{F}}^{-T}}{\partial \boldsymbol{\varphi}} \cdot \Delta \boldsymbol{\varphi} \\
& -\frac{\partial J}{\partial \boldsymbol{\varphi}} \cdot \Delta \boldsymbol{\varphi} B p^{f(k-1)} \hat{\boldsymbol{F}}_{(i)}^{-T}-J_{(i)} B p^{f(k-1)} \frac{\partial \underline{\hat{\boldsymbol{F}}}^{-T}}{\partial \boldsymbol{\varphi}} \cdot \Delta \boldsymbol{\varphi} \\
= & J_{(i)} B^{2} M\left(\ln J_{(i)}-\ln J^{(k-1)}-\frac{p^{f(k-1)}}{B M}\right) \underline{\hat{\boldsymbol{F}}}_{(i)}^{-T} \\
& +J_{(i)} B^{2} M \frac{\partial \ln J}{\partial \boldsymbol{\varphi}} \cdot \Delta \boldsymbol{\varphi} \underline{\hat{\boldsymbol{F}}}_{(i)}^{-T} \\
& +B^{2} M\left(\ln J_{(i)}-\ln J^{(k-1)}-\frac{p^{f(k-1)}}{B M}\right) \frac{\partial J}{\partial \boldsymbol{\varphi}} \cdot \Delta \boldsymbol{\varphi} \underline{\boldsymbol{F}}_{(i)}^{-T} \\
& +J_{(i)} B^{2} M\left(\ln J_{(i)}-\ln J^{(k-1)}-\frac{p^{f(k-1)}}{B M}\right) \frac{\partial \hat{\boldsymbol{F}}^{-T}}{\partial \boldsymbol{\varphi}} \cdot \Delta \boldsymbol{\varphi} .
\end{aligned}
$$

The required derivatives of $J$ and $\ln J$ are obtained by using the chain rule

$$
\begin{gathered}
\frac{\partial J}{\partial \boldsymbol{\varphi}}=\frac{\partial J}{\partial \underline{\hat{\boldsymbol{F}}}}: \frac{\partial \underline{\hat{\boldsymbol{F}}}}{\partial \boldsymbol{\varphi}}, \\
\frac{\partial \ln J}{\partial \boldsymbol{\varphi}}=\frac{\partial \ln J}{\partial J} \frac{\partial J}{\partial \underline{\hat{\boldsymbol{F}}}}: \frac{\partial \hat{\boldsymbol{F}}}{\partial \varphi}
\end{gathered}
$$

and with $\partial J / \partial \underline{\hat{\boldsymbol{F}}}=J \underline{\hat{\boldsymbol{F}}}^{-T}[30]$ as

$$
\begin{gathered}
\frac{\partial J}{\partial \varphi}=J \underline{\hat{\boldsymbol{F}}}^{-T}: \frac{\partial \hat{\boldsymbol{F}}}{\partial \boldsymbol{\varphi}} \\
\frac{\partial \ln J}{\partial \varphi}=\frac{1}{J} J \underline{\hat{\boldsymbol{F}}}^{-T}: \frac{\partial \hat{\boldsymbol{F}}}{\partial \boldsymbol{\varphi}}=\underline{\hat{\boldsymbol{F}}}^{-T}: \frac{\partial \hat{\boldsymbol{F}}}{\partial \boldsymbol{\varphi}} .
\end{gathered}
$$

Using index notation, the relation

$$
\left[\frac{\partial \underline{\hat{\boldsymbol{F}}}}{\partial \boldsymbol{\varphi}} \cdot \Delta \boldsymbol{\varphi}\right]_{i j}=\frac{\partial \hat{F}_{i j}}{\partial \varphi_{k}} \cdot \Delta \varphi_{k}=\frac{\partial^{2} \varphi_{i}}{\partial \chi_{j} \partial \varphi_{k}} \cdot \Delta \varphi_{k}=\frac{\partial}{\partial \chi_{j}} \delta_{i k} \cdot \Delta \varphi_{k}=\frac{\partial \Delta \varphi_{i}}{\partial \chi_{j}}=[\operatorname{Grad} \Delta \varphi]_{i j}
$$


is obtained. Further, the derivative of the inverse and transposed deformation gradient $\hat{\boldsymbol{F}}^{-T}$ with respect to $\varphi$ is required. It is obtained by using the relations $\partial \underline{A}^{-1} / \partial \underline{\boldsymbol{A}}: \underline{\boldsymbol{B}}=-\underline{\boldsymbol{A}}^{-1} \cdot \underline{\boldsymbol{B}} \cdot \underline{A}^{-1}$ as well as $\partial \underline{\boldsymbol{A}}^{T} / \partial \underline{\boldsymbol{A}}: \underline{\boldsymbol{B}}=\underline{\boldsymbol{B}}^{T}$ given in [30] as

$$
\begin{aligned}
\frac{\partial \hat{\boldsymbol{F}}^{-T}}{\partial \varphi} \cdot \Delta \varphi & =\frac{\partial \hat{\boldsymbol{F}}^{-T}}{\partial \underline{\boldsymbol{F}}^{T}}: \frac{\partial \underline{\boldsymbol{F}}^{T}}{\partial \hat{\boldsymbol{F}}}: \frac{\partial \hat{\boldsymbol{F}}}{\partial \boldsymbol{\varphi}} \cdot \Delta \varphi \\
& =-\underline{\hat{\boldsymbol{F}}}^{-T} \cdot\left[\frac{\partial \underline{\hat{\boldsymbol{F}}}^{T}}{\partial \underline{\hat{\boldsymbol{F}}}}: \frac{\partial \hat{\boldsymbol{F}}}{\partial \boldsymbol{\varphi}} \cdot \Delta \boldsymbol{\varphi}\right] \cdot \underline{\hat{\boldsymbol{F}}}^{-T} \\
& =\underline{\hat{\boldsymbol{F}}}^{-T} \cdot\left[\frac{\partial \underline{\hat{\boldsymbol{F}}}}{\partial \boldsymbol{\varphi}} \cdot \Delta \boldsymbol{\varphi}\right]^{T} \cdot \underline{\hat{\boldsymbol{F}}}^{-T}=\underline{\hat{\boldsymbol{F}}}^{-T} \cdot[\operatorname{Grad} \Delta \boldsymbol{\varphi}]^{T} \cdot \underline{\boldsymbol{F}}^{-T}
\end{aligned}
$$

The linearization of the internal forces in the weak form for the undrained split can then be written by introducing $A_{(i)}=B^{2} M\left(\ln J_{(i)}-\ln J^{(k-1)}-p^{f(k-1)} / B M\right)$ as

$$
\begin{aligned}
& \int_{\chi(B)} \underline{\hat{\boldsymbol{P}}}_{(i+1)}: \operatorname{Grad} \boldsymbol{\eta} \mathrm{d} \hat{V}=\int_{\chi(B)}\left(\underline{\hat{\boldsymbol{F}}} \cdot \underline{\hat{\boldsymbol{S}}}^{\prime}\right)_{(i+1)}: \operatorname{Grad} \boldsymbol{\eta} \mathrm{d} \hat{V} \\
& +\int_{\chi(B)}\left(\underline{\hat{\boldsymbol{P}}}^{f \star}\right)_{(i+1)}: \operatorname{Grad} \boldsymbol{\eta} \mathrm{d} \hat{V} \\
& =\int_{\chi(B)}\left(\underline{\hat{\boldsymbol{F}}} \cdot \underline{\hat{\boldsymbol{S}}}^{\prime}\right)_{(i)}: \operatorname{Grad} \boldsymbol{\eta} \mathrm{d} \hat{V} \\
& +\int_{\chi(B)}\left(\Delta \underline{\hat{\boldsymbol{F}}} \cdot \underline{\hat{\boldsymbol{S}}}_{(i)}^{\prime}+\underline{\hat{\boldsymbol{F}}}_{(i)} \cdot \Delta \underline{\hat{\boldsymbol{S}}}^{\prime}\right): \operatorname{Grad} \boldsymbol{\eta} \mathrm{d} \hat{V} \\
& +\int_{\chi(B)} J_{(i)} A_{(i)} \underline{\hat{\boldsymbol{F}}}_{(i)}^{-T}: \operatorname{Grad} \boldsymbol{\eta} \mathrm{d} \hat{V} \\
& +\int_{\chi(B)}\left[J_{(i)} B^{2} M \hat{\boldsymbol{F}}_{(i)}^{-T}: \operatorname{Grad} \boldsymbol{\eta}\right]\left[\underline{\hat{\boldsymbol{F}}}_{(i)}^{-T}: \operatorname{Grad} \Delta \varphi\right] \mathrm{d} \hat{V} \\
& +\int_{\chi(B)}\left[A_{(i)} \underline{\hat{\boldsymbol{F}}}_{(i)}^{-T}: \operatorname{Grad} \boldsymbol{\eta}\right]\left[J_{(i)} \underline{\hat{\boldsymbol{F}}}_{(i)}^{-T}: \operatorname{Grad} \Delta \boldsymbol{\varphi}\right] \mathrm{d} \hat{V} \\
& +\int_{\chi(B)} J_{(i)} A_{(i)}\left[\underline{\hat{\boldsymbol{F}}}^{-T} \cdot[\operatorname{Grad} \Delta \boldsymbol{\varphi}]^{T} \cdot \underline{\hat{\boldsymbol{F}}}^{-T}\right]: \operatorname{Grad} \boldsymbol{\eta} \mathrm{d} \hat{V} .
\end{aligned}
$$

3.1.3. Semi-drained split. In the third case, the predictor of the undrained split is modified as follows:

$$
B f_{\text {semi }} \frac{\dot{J}}{J}+\frac{1}{M} \dot{p}^{f}=0
$$

where

$$
f_{\text {semi }}=\frac{1}{1+\log \frac{K_{\text {mix }}}{\left(1-\phi^{f}\right) K}}
$$

and

$$
K_{\mathrm{mix}}=\left(1-\phi^{f}\right) K+\phi^{f} K_{f}
$$


Then, the predictor is obtained analogously to the undrained split as

$$
p_{\text {semi }}^{\star}=-M B f_{\text {semi }}\left(\ln J^{(k)}-\ln J^{(k-1)}\right)+p^{f(k-1)},
$$

where $k$ indicates the step of the staggered solid-fluid iteration. For the linearization of the balance of momentum with respect to $\varphi$, the first Piola-Kirchhoff stress is then replaced by $\underline{\hat{\boldsymbol{P}}}=\underline{\hat{\boldsymbol{F}}} \cdot \underline{\hat{\boldsymbol{S}}^{\prime}}+\underline{\hat{\boldsymbol{P}}}_{\text {semi }}^{f \star}$ with

$$
\underline{\hat{\boldsymbol{P}}}_{\mathrm{semi}}^{f \star}=J^{(k)} B^{2} M f_{\mathrm{semi}}\left(\ln J^{(k)}-\ln J^{(k-1)}\right) \underline{\hat{\boldsymbol{F}}}^{(k)^{-T}}-J^{(k)} B p^{f(k-1)} \underline{\hat{\boldsymbol{F}}}^{(k)^{-T}}
$$

\subsection{Weak form and linearization of balance of mass}

Applying Galerkin's method, the weak form of the balance of mass for compressible constituents is derived as

$$
\int_{\chi(B)} \psi B \frac{\dot{J}}{J} \mathrm{~d} \hat{V}+\int_{\chi(B)} \psi \frac{1}{M} \dot{p}^{f} \mathrm{~d} \hat{V}-\int_{\chi(B)} \psi \nabla^{\chi} \cdot\left(\underline{\hat{\boldsymbol{K}}} \cdot \nabla^{\chi} p^{f}\right) \mathrm{d} \hat{V}=0 .
$$

Using the product rule for divergence and the Gauss theorem, it reads

$$
\begin{aligned}
0= & \int_{\chi(B)} \psi B \frac{\dot{J}}{J} \mathrm{~d} \hat{V}+\int_{\chi(B)} \psi \frac{1}{M} \dot{p}^{f} \mathrm{~d} \hat{V}+\int_{\chi(B)} \nabla^{\chi} \psi \cdot\left(\underline{\hat{\boldsymbol{K}}} \cdot \nabla^{\chi} p^{f}\right) \mathrm{d} \hat{V} \\
& -\int_{\partial \chi(B)} \psi \underline{\hat{\boldsymbol{K}}} \cdot \nabla^{\chi} p^{f} \cdot \hat{\boldsymbol{N}} \mathrm{d} \hat{\mathrm{A}} \\
= & H\left(\varphi, p^{f}, \psi\right) .
\end{aligned}
$$

The time derivative of the Jacobian $J$ in the reference frame is given with Equation (4) by

$$
\dot{J}=\left.\frac{\partial J}{\partial t}\right|_{\chi}+\nabla^{\chi} J \cdot w
$$

and simplifies for the steady state case to

$$
\dot{J}=\nabla^{\chi} J \cdot \boldsymbol{w} .
$$

Further, the time derivative of $p^{f}$ is required. It is obtained analogously by

$$
\dot{p}^{f}=\left.\frac{\partial p^{f}}{\partial t}\right|_{\chi}+\nabla^{\chi} p^{f} \cdot \boldsymbol{w}
$$

and for the steady-state case by

$$
\dot{p}^{f}=\nabla^{x} p^{f} \cdot \boldsymbol{w} .
$$

For the numerical solution, Equation (65) is linearized as $H \approx H^{k}+\delta H$. Because for the solution of the balance of mass, the deformation is fixed $\varphi=\varphi^{(k)}=$ const. in the staggered iteration scheme (so-called fixed strain split), $p^{f}$ remains the only variable value, so that the linearization is then given by

$$
\begin{aligned}
& \int_{\chi(B)} \nabla^{\chi} \psi \cdot\left(\underline{\hat{\boldsymbol{K}}}^{(k)} \cdot \nabla^{\chi} \delta p^{f}\right) \mathrm{d} \hat{V}+\int_{\chi(B)} \psi \frac{1}{M^{(k)}} \nabla^{\chi} \delta p^{f} \cdot \boldsymbol{w} \mathrm{d} \hat{V} \\
= & \int_{\partial \chi(B)} \psi \underline{\hat{\boldsymbol{K}}}^{(k)} \cdot \nabla^{\chi} p^{f(k-1)} \cdot \hat{\boldsymbol{N}} \mathrm{d} \hat{\mathrm{A}}-\int_{\chi(B)} \psi \frac{B}{J^{(k)}} \nabla^{\chi} J^{(k)} \cdot \boldsymbol{w} \mathrm{d} \hat{V} \\
& -\int_{\chi(B)} \nabla^{\chi} \psi \cdot\left(\underline{\hat{\boldsymbol{K}}}^{(k)} \cdot \nabla^{\chi} p^{f(k-1)}\right) \mathrm{d} \hat{V}-\int_{\chi(B)} \psi \frac{1}{M^{(k)}} \nabla^{\chi} p^{f(k-1)} \cdot \boldsymbol{w} \mathrm{d} \hat{V}
\end{aligned}
$$

with $p^{f(k)}=p^{f(k-1)}+\delta p^{f}, J^{k}=J\left(\varphi^{k}\right)$ and $\underline{\hat{\boldsymbol{K}}}^{k}=\underline{\hat{\boldsymbol{K}}}\left(\boldsymbol{\varphi}^{k}\right)$. It is worth noting that the mass balance leads to a linear system of equations, which requires no further local iteration. 


\subsection{Discretization of the balance of momentum}

Introducing matrix $\underline{\boldsymbol{H}}$, which includes the shape functions $N_{n}\left(\xi_{1}, \xi_{2}, \xi_{3}\right)$ for each node $n$, and vectors $\tilde{\boldsymbol{a}}^{T}=\left(\ldots, a_{1}^{(n)}, a_{2}^{(n)}, a_{3}^{(n)}, \ldots\right)$ of the nodal values at node $n$, the discretizations

$$
\chi=\underline{\boldsymbol{H}} \tilde{\chi}, \quad \boldsymbol{\varphi}=\underline{\boldsymbol{H}} \tilde{\boldsymbol{\varphi}}, \quad \delta \boldsymbol{\varphi}=\underline{\boldsymbol{H}} \delta \tilde{\boldsymbol{\varphi}} \quad \text { and } \quad \boldsymbol{\eta}=\underline{\boldsymbol{H}} \tilde{\boldsymbol{\eta}}
$$

are obtained. Further, the discretization of Grad $\Delta \varphi$ and Grad $\eta$ is given in vector form by

$$
\operatorname{Grad}_{\mathrm{v}} \delta \boldsymbol{\varphi}=\underline{\boldsymbol{H}}_{, \chi} \delta \tilde{\boldsymbol{\varphi}} \quad \text { and } \quad \operatorname{Grad}_{\mathrm{v}} \boldsymbol{\eta}=\underline{\boldsymbol{H}}_{, \chi} \tilde{\boldsymbol{\eta}}
$$

with $\left[\operatorname{Grad}_{\mathrm{v}} \boldsymbol{a}\right]_{k}=[\operatorname{Grad} \boldsymbol{a}]_{i j}$ for $k=3(i-1)+j$. Switching to Voigt notation, the discretized form of the linearized internal work for the drained split is given by

$$
\begin{aligned}
& \tilde{\boldsymbol{\eta}}^{T}\left(\underline{\boldsymbol{K}}_{\mathrm{dr}(i)}^{e} \Delta \tilde{\boldsymbol{\varphi}}^{e}+\hat{\boldsymbol{f}}_{\mathrm{dr} \sigma(i)}^{e}\right) \\
= & \tilde{\boldsymbol{\eta}}^{T} \int_{\chi\left(B^{e}\right)}\left(\underline{\hat{\boldsymbol{B}}}_{(i)}^{T} \hat{\boldsymbol{\mathcal { \boldsymbol { c } }}}_{(i)} \underline{\hat{\boldsymbol{B}}}_{(i)}+\underline{\boldsymbol{H}}_{, \chi}^{T} \underline{\tilde{\boldsymbol{S}}}_{(i)} \underline{\boldsymbol{H}}_{, \chi}\right) \mathrm{d} \hat{V} \Delta \tilde{\boldsymbol{\varphi}}^{e}+\tilde{\boldsymbol{\eta}}^{T} \int_{\chi\left(B^{e}\right)} \underline{\hat{\boldsymbol{B}}}_{(i)}^{T} \hat{\boldsymbol{S}}_{(i)} \mathrm{d} \hat{V}
\end{aligned}
$$

with the material tangent in Voigt notation $\hat{\mathcal{C}}_{(i)}$ and the matrix $\underline{\hat{\boldsymbol{B}}}_{(i)}$ analogously to a standard Lagrangian formulation. Using relation (25), the current drained element stiffness matrix $\underline{\boldsymbol{K}}_{\mathrm{dr}(i)}^{e}$ is obtained in terms of the effective second Piola-Kirchhoff stress tensor and in terms of the fixed $\underline{\hat{S}}^{f(k-1)}$ in dependence on the pore pressure as

$$
\begin{aligned}
& \underline{\boldsymbol{K}}_{\mathrm{dr}(i)}^{e}=\int_{\chi\left(B^{e}\right)} \underline{\hat{\boldsymbol{B}}}_{(i)}^{T} \hat{\boldsymbol{\mathcal { C }}}_{(i)} \hat{\boldsymbol{B}}_{(i)} \mathrm{d} \hat{V} \\
& +\int_{\chi\left(B^{e}\right)} \underline{\boldsymbol{H}}_{, \chi}^{T}{\underline{\boldsymbol{S}^{\prime}}}_{(i)} \underline{\boldsymbol{H}}_{, \chi} \mathrm{d} \hat{V}+\int_{\chi\left(B^{e}\right)} \underline{\boldsymbol{H}}_{, \chi}^{T} \underline{\tilde{\boldsymbol{S}}}^{f(k-1)} \underline{\boldsymbol{H}}_{, \chi} \mathrm{d} \hat{V}
\end{aligned}
$$

and the vector of internal stresses is split into

$$
\hat{\boldsymbol{f}}_{\mathrm{dr} \sigma(i)}^{e}=\int_{\chi\left(B^{e}\right)} \underline{\hat{\boldsymbol{B}}}_{(i)}^{T} \hat{\boldsymbol{S}}_{(i)}^{\prime} \mathrm{d} \hat{V}+\int_{\chi\left(B^{e}\right)} \underline{\hat{\boldsymbol{B}}}_{(i)}^{T} \hat{\boldsymbol{S}}^{f(k-1)} \mathrm{d} \hat{V} .
$$

For the discretization of the undrained split, the vector $\hat{\boldsymbol{F}}_{\mathrm{iT}}$ with $\left[\hat{\boldsymbol{F}}_{\mathrm{iT}}\right]_{k}=\left[\hat{\boldsymbol{F}}^{-T}\right]_{i j}$ with $k=3(i-1)+j$ as well as $\left[\hat{\boldsymbol{F}}_{\mathrm{iTiT}}\right]_{k l}=-\left[\underline{\hat{\boldsymbol{F}}}^{-T}\right]_{i j}\left[\hat{\boldsymbol{F}}^{-T}\right]_{m n}$ with $k=3(i-1)+n$ and $l=3(m-1)+j$ are introduced. Subsequently, the discretized form of (58) might be written as

$$
\begin{aligned}
& \tilde{\boldsymbol{\eta}}^{T}\left(\underline{\boldsymbol{K}}_{\mathrm{un}(i)}^{e} \Delta \tilde{\boldsymbol{\varphi}}^{e}+\hat{\boldsymbol{f}}_{\mathrm{un} \sigma(i)}^{e}\right) \\
& =\tilde{\boldsymbol{\eta}}^{T} \int_{\chi\left(B^{e}\right)}\left(\underline{\hat{\boldsymbol{B}}}_{(i)}^{T} \underline{\hat{\boldsymbol{c}}}_{(i)} \underline{\hat{\boldsymbol{B}}}_{(i)}+\underline{\boldsymbol{H}}_{, \chi}^{T}{\underline{\tilde{\boldsymbol{S}}^{\prime}}}_{(i)} \underline{\boldsymbol{H}}_{, \chi}\right) \mathrm{d} \hat{V} \Delta \tilde{\boldsymbol{\varphi}}^{e} \\
& +\tilde{\boldsymbol{\eta}}^{T} \int_{\chi\left(B^{e}\right)}\left(J_{(i)} A_{(i)}+J_{(i)} B^{2} M\right) \underline{\boldsymbol{H}}_{, \chi}^{T} \hat{\boldsymbol{F}}_{\mathrm{iT}(i)} \hat{\boldsymbol{F}}_{\mathrm{iT}(i)}^{T} \underline{\boldsymbol{H}}_{, \chi} \mathrm{d} \hat{V} \Delta \tilde{\boldsymbol{\varphi}}^{e} \\
& +\tilde{\boldsymbol{\eta}}^{T} \int_{\chi\left(B^{e}\right)}\left(J_{(i)} A_{(i)}\right) \underline{\boldsymbol{H}}_{, \chi}^{T} \hat{\boldsymbol{\boldsymbol { F }}}_{\text {iTiT (i) }} \underline{\boldsymbol{H}}_{, \chi} \mathrm{d} \hat{V} \Delta \tilde{\boldsymbol{\varphi}}^{e} \\
& +\tilde{\boldsymbol{\eta}}^{T} \int_{\chi\left(B^{e}\right)} \underline{\hat{\boldsymbol{B}}}_{(i)}^{T} \hat{\boldsymbol{S}}_{(i)}^{\prime} \mathrm{d} \hat{V} \\
& +\tilde{\boldsymbol{\eta}}^{T} \int_{\chi\left(B^{e}\right)}\left(J_{(i)} A_{(i)}\right) \underline{\boldsymbol{H}}_{, \chi}^{T} \hat{\boldsymbol{F}}_{\mathrm{iT}(i)} \mathrm{d} \hat{V} .
\end{aligned}
$$


The discretization of the external forces is similar to the Lagrangian formulation and reads in the ALE frame

$$
\tilde{\boldsymbol{\eta}}^{T}\left(\hat{\boldsymbol{f}}_{\mathrm{ext}}^{e}\right)=\tilde{\boldsymbol{\eta}}^{T}\left(\int_{\chi\left(B^{e}\right)} \hat{\rho} \underline{\boldsymbol{H}}^{T} \boldsymbol{G} \mathrm{d} \hat{V}+\int_{\partial \chi\left(B^{e}\right)} \underline{\boldsymbol{H}}^{T} \overline{\boldsymbol{T}} \mathrm{d} \hat{\mathrm{A}}\right) .
$$

For the discretization of the linearized virtual work of inertia forces, the matrix

$$
\underline{\boldsymbol{A}}=\left[\begin{array}{ccccccc}
N_{1, i} w_{i} & 0 & 0 & \ldots & N_{n_{k}, i} w_{i} & 0 & 0 \\
0 & N_{1, i} w_{i} & 0 & \ldots & 0 & N_{n_{k}, i} w_{i} & 0 \\
0 & 0 & N_{1, i} w_{i} & \ldots & 0 & 0 & N_{n_{k}, i}, w_{i}
\end{array}\right]
$$

is introduced [8], where $N_{k, i} w_{i}:=\sum_{i}\left(N_{k, i} w_{i}\right)$. With

$$
\operatorname{Grad} \boldsymbol{\varphi} \cdot \boldsymbol{w}=\underline{\boldsymbol{A}} \tilde{\boldsymbol{\varphi}} \quad \text { and } \operatorname{Grad} \Delta \boldsymbol{\varphi} \cdot \boldsymbol{w}=\underline{\boldsymbol{A}} \Delta \tilde{\boldsymbol{\varphi}}
$$

the linearized and discretized formulation of the inertia term is obtained as

$$
\begin{aligned}
& \tilde{\boldsymbol{\eta}}^{T}\left(-\underline{\boldsymbol{W}}^{e} \Delta \tilde{\boldsymbol{\varphi}}^{e}+\hat{\boldsymbol{f}}_{\mathrm{i}(i)}^{e}\right) \\
= & \tilde{\boldsymbol{\eta}}^{T}\left(\int_{\chi\left(B^{e}\right)} \hat{\rho} \underline{\boldsymbol{A}}^{T} \underline{\boldsymbol{A}} \mathrm{d} \hat{V}-\int_{\partial \chi\left(B^{e}\right)} \hat{\rho} \underline{\boldsymbol{H}}^{T} \underline{\boldsymbol{A}}(\boldsymbol{w} \cdot \hat{\boldsymbol{N}}) \mathrm{d} \hat{\mathrm{A}}\right) \Delta \tilde{\boldsymbol{\varphi}}^{e} \\
& +\tilde{\boldsymbol{\eta}}^{T}\left(\int_{\chi\left(B^{e}\right)} \hat{\rho} \underline{\boldsymbol{A}}^{T} \underline{\boldsymbol{A}} \tilde{\boldsymbol{\varphi}}_{(i)}^{e} \mathrm{~d} \hat{V}-\int_{\partial \chi\left(B^{e}\right)} \hat{\rho} \underline{\boldsymbol{H}}^{T} \underline{\boldsymbol{A}}_{\tilde{\boldsymbol{\varphi}}_{(i)}^{e}}(\boldsymbol{w} \cdot \hat{\boldsymbol{N}}) \mathrm{d} \hat{\mathrm{A}}\right) .
\end{aligned}
$$

By assembling over all finite elements, the linearized system of equations of momentum balance for a steady state rolling or moving body is obtained

$$
\left(\underline{\boldsymbol{K}}_{(i)}-\underline{\boldsymbol{W}}\right) \Delta \tilde{\boldsymbol{\varphi}}=\hat{\boldsymbol{f}}_{\mathrm{ext}}-\hat{\boldsymbol{f}}_{\sigma(i)}-\hat{\boldsymbol{f}}_{\mathrm{i}(i)}
$$

Because of the steady state formulation in the reference frame, the system of equations (81) is time independent, although inertia effects are considered, so that in contrast to a Lagrangian computation, no time step algorithm is required.

\subsection{Discretization of the balance of mass}

For the discretization of the scalar-valued pore pressure and test function

$$
p^{f}=\boldsymbol{h}^{T} \tilde{\boldsymbol{p}}^{f}, \quad \delta p^{f}=\boldsymbol{h}^{T} \delta \tilde{\boldsymbol{p}}^{f} \quad \text { and } \quad \psi=\boldsymbol{h}^{T} \tilde{\boldsymbol{\psi}}
$$

a vector $\boldsymbol{h}$, which contains the nodal shape functions, and vectors of the nodal values $\tilde{\boldsymbol{a}}^{T}=$ $\left(\ldots, a^{(n)}, \ldots\right)$ at node $n$ are introduced. Subsequently, the corresponding gradients might be expressed as 


$$
\begin{aligned}
\nabla^{\chi} p^{f}\left(\xi_{1}, \xi_{2}, \xi_{3}\right) & =\underline{\boldsymbol{h}},{ }_{\chi} \tilde{\boldsymbol{p}}^{f}, \\
\nabla^{\chi} \delta p^{f}\left(\xi_{1}, \xi_{2}, \xi_{3}\right) & =\underline{\boldsymbol{h}}{ }_{\chi} \delta \tilde{\boldsymbol{p}}^{f}, \\
\nabla^{\chi} \psi\left(\xi_{1}, \xi_{2}, \xi_{3}\right) & =\underline{\boldsymbol{h}},{ }_{\chi} \tilde{\boldsymbol{\psi}} .
\end{aligned}
$$

The discretization of the linearized mass balance (70) requires further the gradient of the Jacobian. In contrast to the pore pressure $p^{f}$ and the test function $\psi$, the Jacobian $J^{(k)}$ is not approximated directly by the shape functions but indirectly via $\chi$ and $\boldsymbol{\varphi}^{(k)}$. The Jacobian is given by

$$
\begin{aligned}
& \boldsymbol{J}^{(k)}\left(\xi_{1}, \xi_{2}, \xi_{3}\right)=\operatorname{det} \underline{\hat{\boldsymbol{F}}}^{(k)}\left(\boldsymbol{\xi}_{1}, \boldsymbol{\xi}_{2}, \boldsymbol{\xi}_{3}\right) \\
& =\hat{F}_{11}^{(k)} \hat{F}_{22}^{(k)} \hat{F}_{33}^{(k)}+\hat{F}_{12}^{(k)} \hat{F}_{23}^{(k)} \hat{F}_{31}^{(k)}+\hat{F}_{13}^{(k)} \hat{F}_{21}^{(k)} \hat{F}_{32}^{(k)} \\
& -\hat{F}_{11}^{(k)} \hat{F}_{23}^{(k)} \hat{F}_{32}^{(k)}-\hat{F}_{12}^{(k)} \hat{F}_{21}^{(k)} \hat{F}_{33}^{(k)}-\hat{F}_{13}^{(k)} \hat{F}_{22}^{(k)} \hat{F}_{31}^{(k)}
\end{aligned}
$$

with

$$
\underline{\hat{\boldsymbol{F}}}^{(k)}\left(\xi_{1}, \xi_{2}, \xi_{3}\right)=\nabla^{\chi} \boldsymbol{\varphi}^{(k)}\left(\xi_{1}, \xi_{2}, \xi_{3}\right) .
$$

The gradient of the Jacobian is obtained as

$$
\begin{aligned}
\nabla^{\chi} J\left(\xi_{1}, \xi_{2}, \xi_{3}\right)= & \nabla^{\chi}\left(\operatorname{det} \hat{\boldsymbol{F}}\left(\xi_{1}, \xi_{2}, \xi_{3}\right)\right) \\
= & \frac{\partial}{\partial \chi}\left(\hat{F}_{11} \hat{F}_{22} \hat{F}_{33}+\hat{F}_{12} \hat{F}_{23} \hat{F}_{31}+\hat{F}_{13} \hat{F}_{21} \hat{F}_{32}\right. \\
& \left.-\hat{F}_{11} \hat{F}_{23} \hat{F}_{32}-\hat{F}_{12} \hat{F}_{21} \hat{F}_{33}+\hat{F}_{13} \hat{F}_{22} \hat{F}_{31}\right) \\
= & \frac{\partial \hat{F}_{11}}{\partial \chi}\left(\hat{F}_{22} \hat{F}_{33}-\hat{F}_{23} \hat{F}_{32}\right)+\frac{\partial \hat{F}_{22}}{\partial \chi}\left(\hat{F}_{11} \hat{F}_{33}-\hat{F}_{13} \hat{F}_{31}\right) \\
& +\frac{\partial \hat{F}_{33}}{\partial \chi}\left(\hat{F}_{11} \hat{F}_{22}-\hat{F}_{12} \hat{F}_{21}\right)+\frac{\partial \hat{F}_{12}}{\partial \chi}\left(\hat{F}_{23} \hat{F}_{31}-\hat{F}_{21} \hat{F}_{33}\right) \\
& +\frac{\partial \hat{F}_{13}}{\partial \chi}\left(\hat{F}_{21} \hat{F}_{32}-\hat{F}_{22} \hat{F}_{31}\right)+\frac{\partial \hat{F}_{21}}{\partial \chi}\left(\hat{F}_{13} \hat{F}_{32}-\hat{F}_{12} \hat{F}_{33}\right) \\
& +\frac{\partial \hat{F}_{23}}{\partial \chi}\left(\hat{F}_{12} \hat{F}_{31}-\hat{F}_{11} \hat{F}_{32}\right)+\frac{\partial \hat{F}_{31}}{\partial \chi}\left(\hat{F}_{12} \hat{F}_{23}-\hat{F}_{13} \hat{F}_{22}\right) \\
& +\frac{\partial \hat{F}_{32}}{\partial \chi}\left(\hat{F}_{13} \hat{F}_{21}-\hat{F}_{11} \hat{F}_{23}\right) .
\end{aligned}
$$

Therefore, the gradient of the components $\hat{F}_{i j}$ of the deformation gradient is required. It is obtained in index notation by using the chain rule

$$
\left[\nabla^{\chi} \hat{F}_{i j}\left(\xi_{1}, \xi_{2}, \xi_{3}\right)\right]_{k}=\frac{\partial^{2} \varphi_{i}}{\partial \chi_{j} \partial \chi_{k}}=\frac{\partial^{2} \varphi_{i}}{\partial \xi_{m} \partial \xi_{n}} \frac{\partial \xi_{m}}{\partial \chi_{j}} \frac{\partial \xi_{n}}{\partial \chi_{k}} .
$$

For the discretization of the second term of the linearized mass balance, the vector

$$
\boldsymbol{A}^{T}=\left(N_{1, i} w_{i}, \ldots, N_{k_{\max }, i} w_{i}\right)
$$

is introduced. Then,

$$
\operatorname{Grad} p^{f} \cdot \boldsymbol{w}=\boldsymbol{A}^{T} \tilde{p}^{f} \quad \text { and } \operatorname{Grad} \delta p^{f} \cdot \boldsymbol{w}=\boldsymbol{A}^{T} \delta \tilde{p}^{f}
$$

are obtained. The balance of mass at fluid-solid iteration step $k$ might then be written as

$$
\underline{\boldsymbol{K}}_{p}^{(k)} \delta \tilde{\boldsymbol{p}}^{f}=\boldsymbol{R}_{\text {ext }}-\boldsymbol{R}_{J}^{(k)}-\boldsymbol{R}_{p}^{(k)}
$$

by assembling over all element matrixes and vectors

$$
\underline{\boldsymbol{K}}_{p}^{(k)}=\cup_{e} \underline{\boldsymbol{K}}_{p}^{e(k)}, \quad \boldsymbol{R}_{e x t}=\cup_{e} \boldsymbol{R}_{e x t}^{e}, \quad \boldsymbol{R}_{J}^{(k)}=\cup_{e} \boldsymbol{R}_{J}^{e(k)}, \quad \boldsymbol{R}_{p}^{(k)}=\cup_{e} \boldsymbol{R}_{p}^{e(k)},
$$


whereby the single element matrixes and vectors are obtained from Equation (70) as

$$
\begin{aligned}
\tilde{\boldsymbol{\psi}}^{T} \underline{\boldsymbol{K}}_{p}^{e(k)} \delta \tilde{\boldsymbol{p}}^{f} & =\tilde{\boldsymbol{\psi}}^{T} \int_{\chi\left(B^{e}\right)}\left(\underline{\boldsymbol{h}}_{\chi}^{T} \underline{\hat{\boldsymbol{K}}}^{(k)} \underline{\boldsymbol{h}}{ }_{\chi}+\frac{1}{M^{(k)}} \boldsymbol{h}^{T} \boldsymbol{A}\right) \mathrm{d} \hat{V} \delta \tilde{\boldsymbol{p}}^{f}, \\
\tilde{\boldsymbol{\psi}}^{T} \boldsymbol{R}_{e x t}^{e} & =\tilde{\boldsymbol{\psi}}^{T} \int_{\partial \chi\left(B^{e}\right)} \boldsymbol{h}^{T}\left(\hat{\boldsymbol{q}}^{f} \cdot \hat{\boldsymbol{N}}\right) \mathrm{d} \hat{\mathrm{A}}, \\
\tilde{\boldsymbol{\psi}}^{T} \boldsymbol{R}_{J}^{e(k)} & =\tilde{\boldsymbol{\psi}}^{T} \int_{\chi\left(B^{e}\right)} \boldsymbol{h}^{T}\left(\frac{B}{\boldsymbol{J}^{(k)}} \nabla^{\chi} \boldsymbol{J}^{(k)} \cdot \boldsymbol{w}\right) \mathrm{d} \hat{V}, \\
\tilde{\boldsymbol{\psi}}^{T} \boldsymbol{R}_{p}^{e(k)} & =\tilde{\boldsymbol{\psi}}^{T} \int_{\chi\left(B^{e}\right)}\left(\underline{\boldsymbol{h}}_{\chi}^{T} \underline{\hat{\boldsymbol{K}}}^{(k)} \underline{\boldsymbol{h}}{ }_{\chi}+\frac{1}{M^{(k)}} \boldsymbol{h}^{T} \boldsymbol{A}\right) \mathrm{d} \hat{V} \tilde{\boldsymbol{p}}^{f(k)} .
\end{aligned}
$$

Please note that $\underline{\boldsymbol{K}}_{p}^{(k)}$ is non-symmetric.

\subsection{Iterative sequential solid-fluid solver}

For the solution of the poromechanical ALE formulation, an iterative solver is chosen. In other words, the residuals of the balance of momentum and the balance of mass are evaluated consecutively until the residuals of both equations are both below the tolerance. To the best knowledge of the authors, this is the first time an operator-split ALE poromechanics solver has been established with a multiplicative kinematics for the finite deformation range. The first operator-split algorithm for poromechanics problems can at least be traced back to [4] in which the author derived an undrained and a drained split operator for finite strain poromechanics problems and found that the drained split may lead to oscillatory iterations. On the other hand, Ehlers et al. [32] analyze the decoupled numerical solution approach and found that the usage of an explicit operator may lead to an iterative multi-physical system being conditionally stable. Similar conclusions were reached by the authors in [33-36] in which fixed stress, fixed strain, undrained, and drained splits are systemically analyzed. Recent works, such as $[37,38]$, provide an interesting re-interpretation of the operator-split schemes and prove that they are equivalent to block-preconditioned Richardson iterations. These results from the analyses indicate that drained and fixed-strain operator splits may lead to only conditionally stable time steps and that the numerical iterations among fluid and solid solvers depend strongly on the strength of the coupling.

Here, we consider three different iterative coupling schemes for the ALE $\mathrm{u} / \mathrm{p}$ formulation, the ALE drained split, the ALE undrained split, and a new split scheme, we refer to as ALE semi-drained split, which are derived based on different assumptions. In all cases, the iterative sequential solver starts with the solid step (evaluation of momentum balance). In case of the drained split, the pore pressure is fixed during the solid step in which the displacements are updated (Table I), while the displacements are fixed during the fluid step, where the pore pressure is updated (evaluation of the mass balance). On the other hand, the ALE undrained split scheme assumes that the fluid content is fixed, which leads to a predictor of the pore pressure $p^{\star}$, while the solid solver updates the displacements (Table II). At the fluid step of this solver, the strain is fixed again while the pore pressure is updated (identical to the drained split solver). The semi-drained solver operates identically to the undrained solver, except for the pore pressure predictor $p_{\text {semi }}^{\star}$, which leads to a modified solid stiffness matrix $\underline{\boldsymbol{K}}_{\mathrm{semi}}$. At all solvers, the residual of the balance of momentum is updated with the new pore pressure $p^{f(k)}$ after the fluid step. If the updated residual is smaller than the prescribed tolerance, the staggered iteration stops. Otherwise, a further sequential iteration step restarts with the solid step.

Analogously to the previous research of, for example, [4, 35], numerical investigations showed that the ALE drained split is only conditionally stable, too. Thus, it is subsequently no more considered. The efficiency of the undrained and semi-drained split is discussed in the transient verification example in the Appendix. The Appendix includes, further, a verification of the poromechanical ALE formulation. 
Table I. Drained iterative sequential fluid-solid solver.

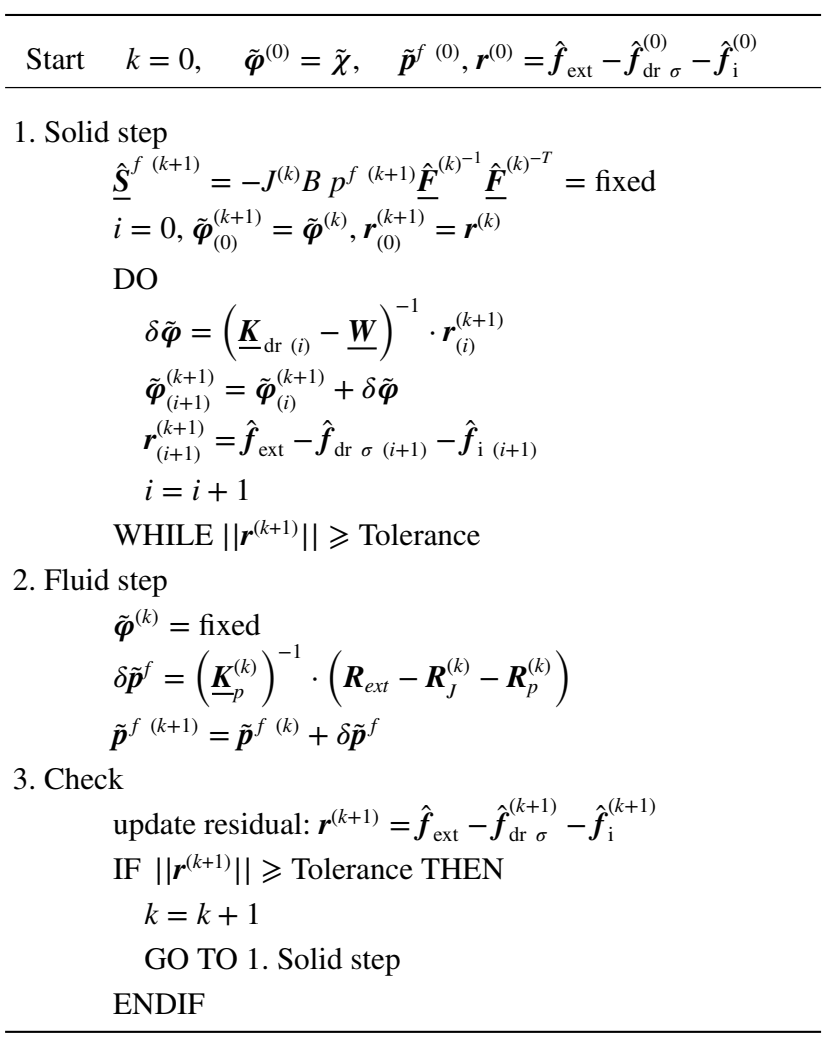

Table II. Undrained iterative sequential fluid-solid solver.

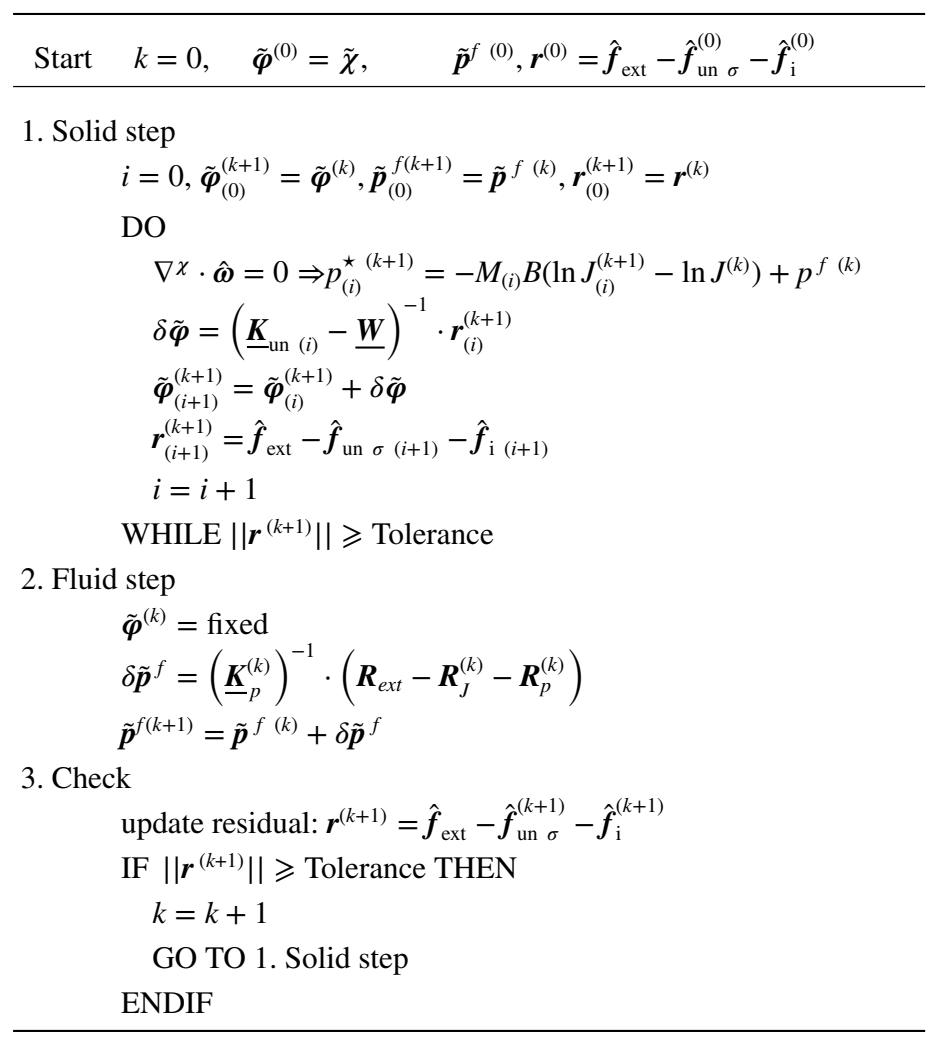




\section{COUPLED TIRE-WATER-SOIL INTERACTION}

\subsection{Hierarchical sequential coupled tire-water-soil computation}

The main goal of the present research is the development of a simulation tool, which enables a realistic representation of tires rolling over a fully saturated soft subground (soil), whereby both contact partners are allowed to be at finite strains. Therefore, the developed poromechanical ALE formulation is implemented into the coupled tire-pavement computation scheme presented in [21]. Because the tire and the pavement are simulated in different FE codes, this tire-pavement computation is organized by a program interface as staggered iteration between the FE ALE tire model, which is able to consider the viscoelastic and temperature-dependent behavior of the tire components [17, 19] at finite strains, and the FE ALE pavement model, which is also formulated for inelastic and temperature dependent material behavior at finite strains [14]. Including the staggered solid-fluid iteration scheme for the poromechanical ALE formulation into the staggered tire-pavement computation scheme demands for a hierarchical solution strategy, where the tire-pavement or rather the tire-soil iteration is the outer iteration loop, while the solid-fluid interaction is solved during an inner iteration loop at the pavement or soil computation (Figure 3). The corresponding flowchart of this hierarchical staggered iteration scheme is illustrated in Figure 4. To simplify the approach for the moment and to concentrate on the poromechanical solid-fluid interaction, the present paper considers a hyperelastic material representation for the tire as well as the soil.

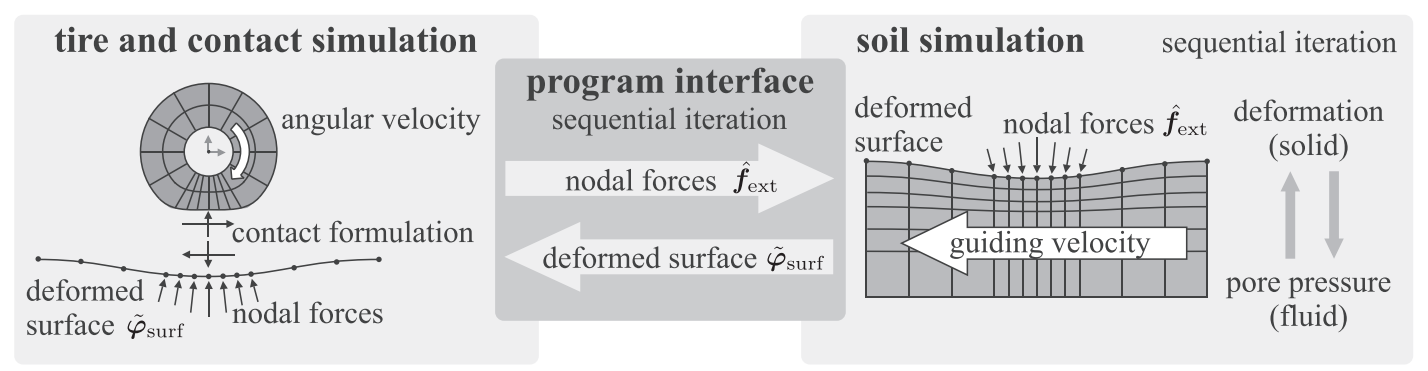

Figure 3. Hierarchical sequential coupling procedure for tire-soil interaction.

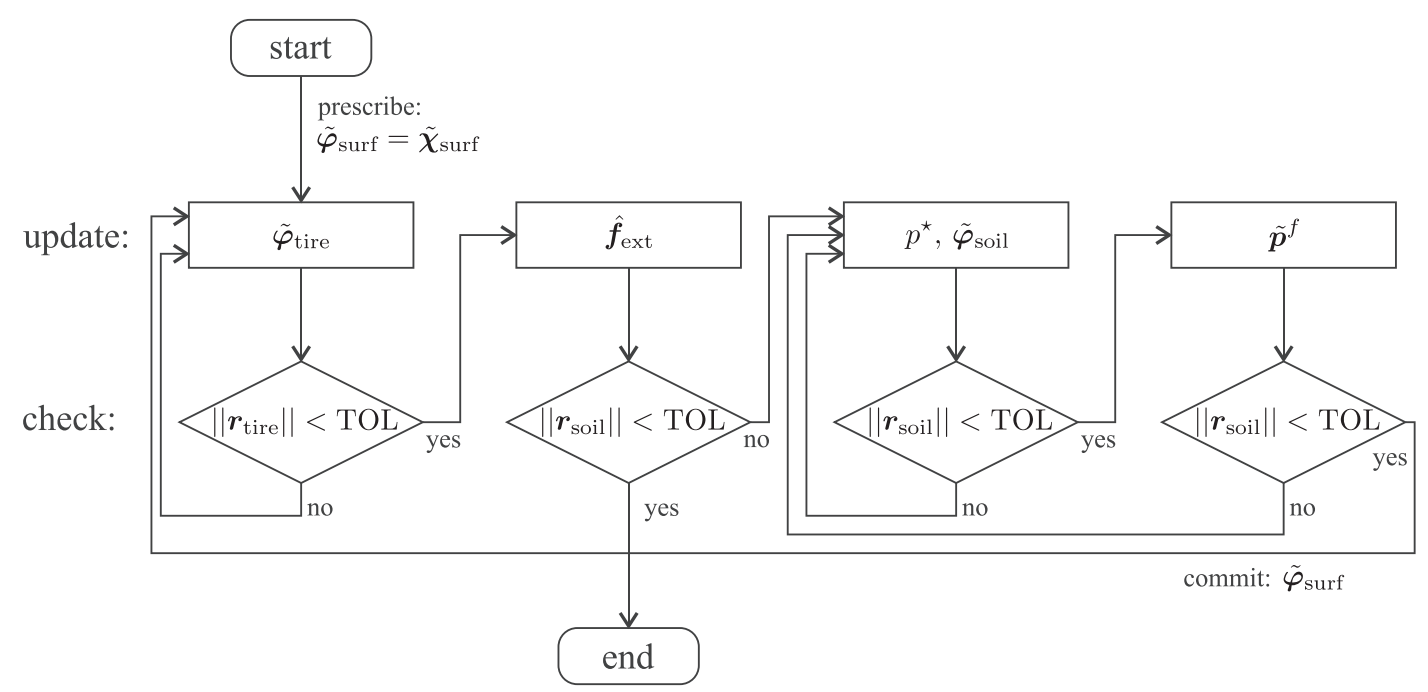

Figure 4. Flowchart of the hierarchical sequential coupling procedure for tire-soil interaction. 
The computation starts with the simulation of the steady state rolling tire on a rigid contact surface, where the shape of the contact surface equals the shape of the pavement or soil surface in the current state. When the residual of the tire becomes smaller than the tolerance, the tire and contact iteration terminates, and the nodal contact forces at the contact surface are transmitted to the soil simulation and used for the update of the external forces $\hat{f}_{\text {ext }}$ as well as of the residual of the soil. As long as the residual of the soil is larger than the tolerance, the iteration scheme proceeds with the iteration of the solid step (update of $p^{\star}$ and $\tilde{\boldsymbol{\varphi}}_{\text {soil }}$ ) and the subsequent fluid step (update of $\tilde{\boldsymbol{p}}^{f}$ ). After the fluid step, the residual of the soil is checked again. If it is larger than the tolerance, a further solid-fluid iteration is performed. Otherwise, the solid-fluid iteration is finished, and the obtained deformation of the soil surface is committed to the next tire and contact iteration. The whole hierarchical staggered iteration scheme ends, when the residual of the soil is still smaller than the tolerance after the update of the external loads subsequently to the tire simulation, which is the case when the resulting nodal contact forces of the current and the previous tire simulation coincide.

\subsection{Rolling truck tire on saturated hyperelastic soil}

The staggered tire-soil iteration scheme is applied for the computation of a hyperelastic truck tire

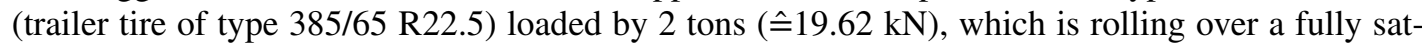
urated soil subground at driving velocities of 1 and $0.5 \mathrm{~m} / \mathrm{s}$, respectively. The solid soil material is represented by the hyperelastic neo-Hookean model with the parameters given in Table III. The FE meshes of the tire and the soil subground are shown in Figure 5. Because of the formulation in the reference frame (ALE formulation), a dense discretization is only required close to the contact area, which is one big advantage of the ALE formulation compared with a transient Lagrangian computation. The tire model considers all relevant structural components (rubber parts, steel cords, textile

Table III. Material parameters of fully saturated soil.

\begin{tabular}{|c|c|c|c|c|}
\hline Solid & $\begin{array}{c}\mu \\
{\left[\mathrm{kN} / \mathrm{m}^{2}\right]}\end{array}$ & $\begin{array}{c}K \\
{\left[\mathrm{kN} / \mathrm{m}^{2}\right]}\end{array}$ & $\begin{array}{c}K_{s} \\
{\left[\mathrm{kN} / \mathrm{m}^{2}\right]}\end{array}$ & $\begin{array}{c}B \\
{[-]}\end{array}$ \\
\hline & 22500 & 40000 & $4 \cdot 10^{8}$ & 1 \\
\hline \multirow[t]{3}{*}{ Fluid } & $k$ & $\hat{\phi}^{f}$ & $K_{f}$ & \\
\hline & {$\left[\mathrm{m}^{4} / \mathrm{kNs}\right]$} & {$[-]$} & {$\left[\mathrm{kN} / \mathrm{m}^{2}\right]$} & \\
\hline & $3.06 \cdot 10^{-6}$ & 0.3 & 80000 & \\
\hline
\end{tabular}

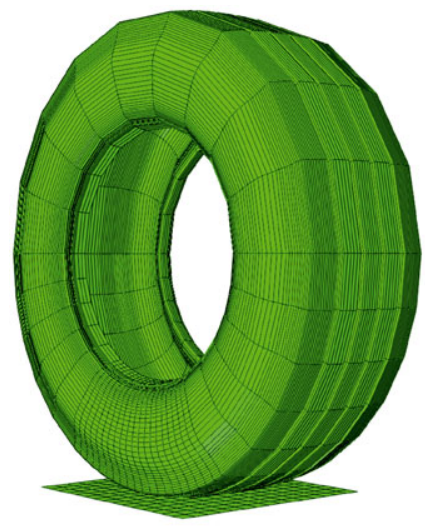

(a)

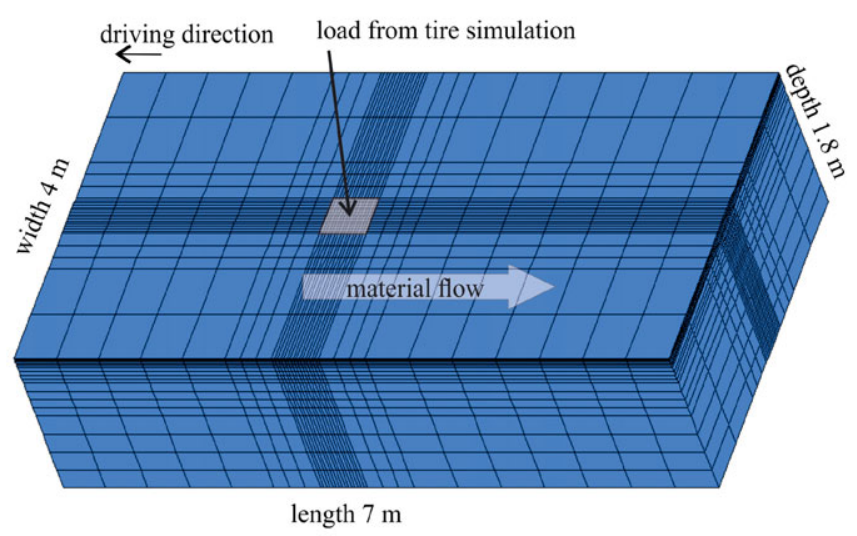

(b)

Figure 5. Finite element meshes of (a) the truck tire and (b) the soil segment. 
reinforcements) by brick and rebar finite elements, respectively. In order to obtain a contact surface, which consists of linear four-node elements (required for contact algorithm), the soil subground is represented by all together 16500 isoparametric eight-node elements (for the solid as well as the fluid part). As boundary conditions, all displacements at the bottom as well as the normal displacements at the lateral surfaces are fixed. The pore pressure is prescribed to zero at the whole top surface, while all other boundaries are undrained $\left(q^{f}=0\right)$.

According to the hierarchical iteration scheme, the computation starts with the tire simulation on the undeformed (flat) contact surface under consideration of Coulomb's friction law with a constant friction coefficient of $\mu_{\text {fric }}=0.9$. The rotational velocity of the tire, which is required to reach the prescribed translational velocity at a certain rolling condition (in this case free rolling), depends on the tire load, the tire structure, and the contact conditions and, therefore, is unknown a priory. It is, thus, determined during each tire simulation process by a user-defined subroutine [39]. The resulting

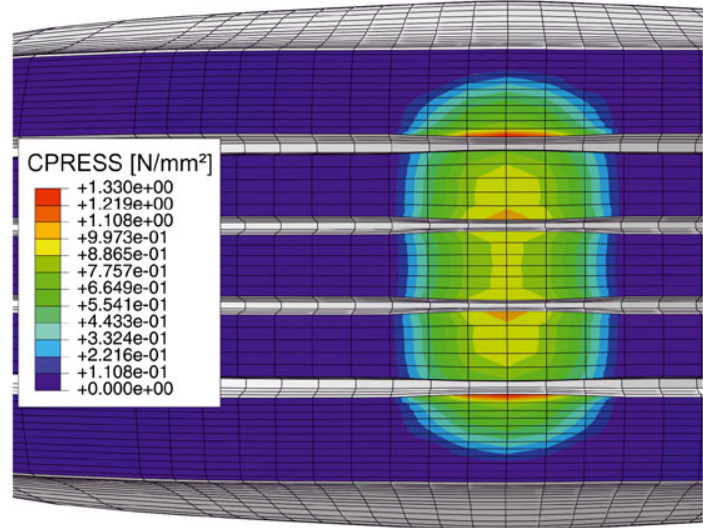

(a)

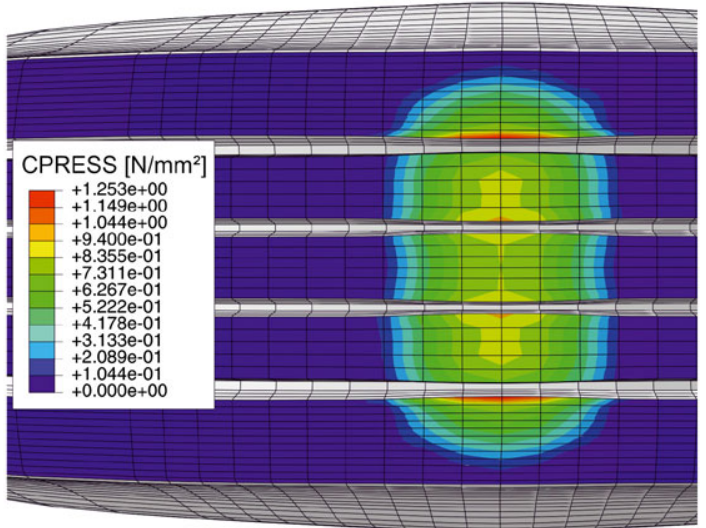

(b)

Figure 6. Contact pressure distribution at the truck tire footprint at $1 \mathrm{~m} / \mathrm{s}$ on (a) the undeformed contact surface and (b) the deformed contact surface.

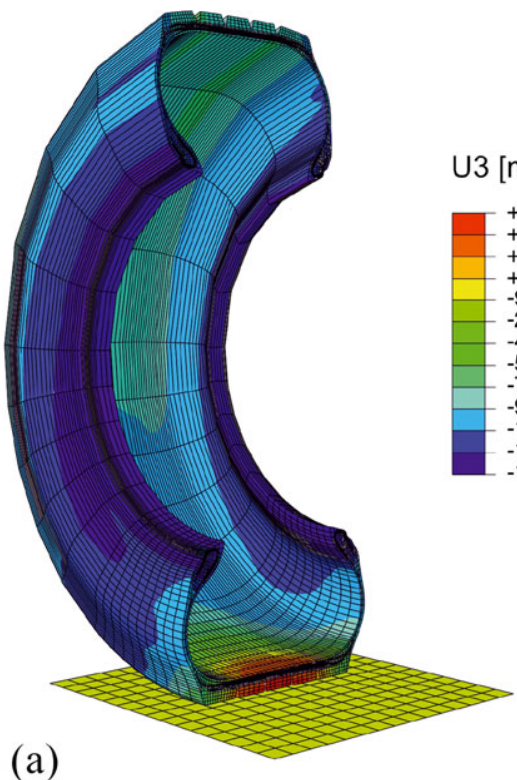

(a)
U3 [mm]

$+5.414 \mathrm{e}+00$ $+3.812 \mathrm{e}+00$ $+2.210 \mathrm{e}+00$ $+6.085 \mathrm{e}-01$ $-9.934 \mathrm{e}-01$ $-2.595 \mathrm{e}+00$ $4.197 \mathrm{e}+00$ $-5.799 \mathrm{e}+00$ $7.401 \mathrm{e}+00$ $-9.003 \mathrm{e}+00$ $1.060 \mathrm{e}+0$ $-1.221 e+01$ $-1.381 \mathrm{e}+01$

Figure 7. Vertical displacements of the truck tire at $1 \mathrm{~m} / \mathrm{s}$ on (a) the undeformed contact surface and (b) the deformed contact surface.

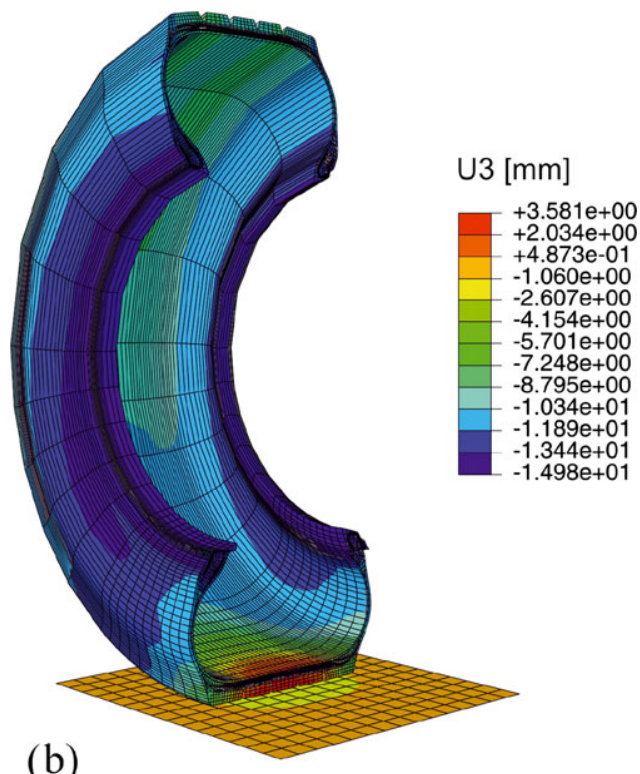

(b) 


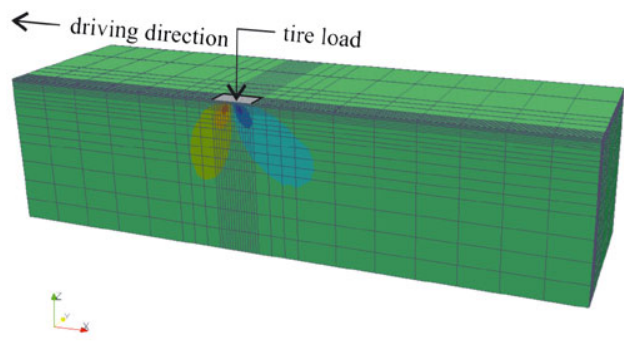

(a)

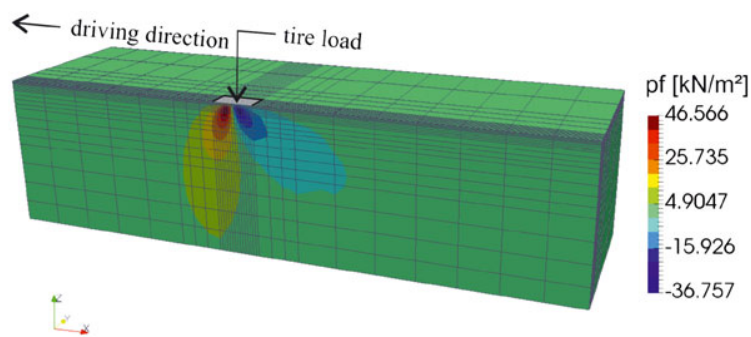

(b)

Figure 8. Pore pressure in plane along driving lane for guiding velocity of (a) $w_{1}=0.5 \mathrm{~m} / \mathrm{s}$ and (b) $w_{1}=$ $1 \mathrm{~m} / \mathrm{s}$.

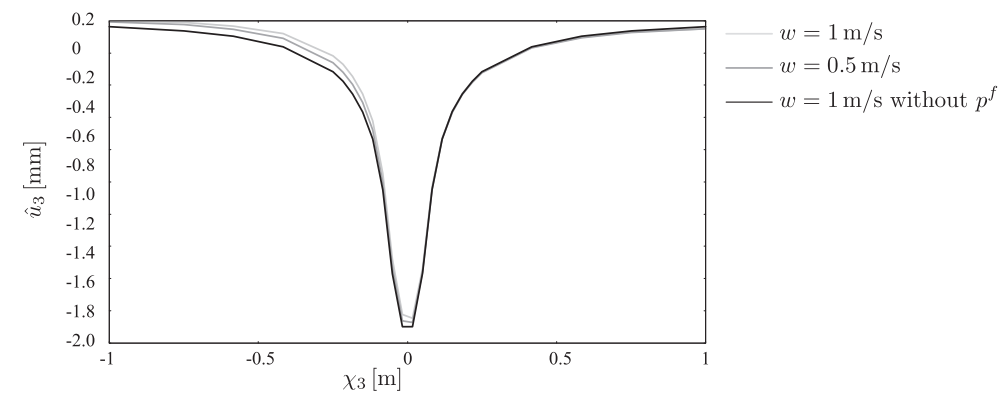

Figure 9. Vertical displacements of the surface along the driving lane for the three computation cases.

contact pressure distribution in the tire footprint on the undeformed contact surface at a translational tire velocity of $1 \mathrm{~m} / \mathrm{s}$ is illustrated in Figure 6 (a). For comparison, Figure 6 (b) shows the final contact pressure distribution on the deformed surface. It can be seen that the contact area increases and the maximum pressure decreases, because of the deformed contact surface. Further, Figure 7 illustrates the vertical displacements of the tire at $1 \mathrm{~m} / \mathrm{s}$ on the (a) undeformed and (b) deformed contact surface. The obtained distribution of the fluid pore pressure in the soil is compared in Figure 8 for the two different speeds. As expected, the pore pressures are smaller at lower speed, because the time for drainage is longer in this case. It is worth noting that there is a region with positive pore pressure in the soil in front of the tire axle (loading) as well as a region with negative pore pressure after the tire axle (unloading). The resulting surface displacements of the saturated soil along the driving lane are given in Figure 9 for the two different speeds as well as for the interaction of the tire with a dry soil (without $p^{f}$ ). It can be seen that the displacements are largest in the dry case, because the solid phase has to carry the whole load in this situation. In case of saturated soil, the displacements decrease with increasing speed because the fluid pore pressure increases and, therefore, the solid effective stress decreases. Further, it can be observed that the non-symmetric distribution of the displacements with respect to the tire axle at $\chi_{3}=0$ increases as well with the driving speed in case of saturated soil.

Summarizing, it can be stated that all obtained results are reasonable, and the influences of existing pore water on the soil behavior as well as on the tire are demonstrated.

\section{CONCLUSION}

This work presents a hierarchical sequential ALE algorithm that captures the tire-soil interaction in which both the tire and the soil are in the finite deformation range. The soil is idealized as a two-phase porous medium consisting of a solid skeleton with distributed pores filled with water. 
In the hierarchical sequential scheme, there are two major interaction mechanisms captured, that is, (1) tire and soil at the contact surface and (2) interaction between the solid skeleton and the pore fluid within the soil. To the best knowledge of the authors, this is the first time in which (1) an ALE iterative solver for the finite strain poromechanics problem has been formulated with multiplicative kinematics and (2) the ALE finite strain poromechanics has been used to capture the tire-soil-water interaction. Main effects of existing pore water on the tire-soil interaction are demonstrated in the numerical example. The presented research is an essential basis for further developments considering, for example, unsaturated soils and inelastic soil models like the Cam-Clay model for a more realistic representation of off-road rolling tires.

\section{APPENDIX}

\section{Transient Lagrangian formulation of mass balance}

The time derivative of the Jacobian $J$ in the Lagrangian frame is given [6] by

$$
\frac{\dot{J}}{J}=(\ln J) \approx \frac{\ln J_{n+1}-\ln J_{n}}{\Delta t}
$$

and the time derivative of $p^{f}$ is approximated analogously by

$$
\dot{p}^{f} \approx \frac{p_{n+1}^{f}-p_{n}^{f}}{\Delta t} .
$$

For a numerical solution, the balance of mass is linearized as $H \approx H^{k}+\delta H$ with respect to the Lagrangian frame. Because for the solution of the balance of mass, the deformation is fixed $\boldsymbol{x}=$ $\boldsymbol{x}^{(k)}=$ const., $p^{f}$ remains the only variable value, so that the linearization is then given by

$$
\begin{aligned}
& \int_{B} \nabla^{X} \psi \cdot\left(\underline{\boldsymbol{K}}^{(k)} \cdot \nabla^{X} \delta p^{f}\right) \mathrm{d} V+\int_{B} \psi \frac{1}{M^{(k)} \Delta t} \delta p^{f} \mathrm{~d} V \\
= & \int_{\partial B} \psi \underline{\boldsymbol{K}}^{(k)} \cdot \nabla^{X} p^{f(k)} \cdot \boldsymbol{N} \mathrm{d} A-\int_{B} \psi B \frac{\ln J_{n+1}^{(k)}-\ln J_{n}}{\Delta t} \mathrm{~d} V \\
& -\int_{B} \nabla^{X} \psi \cdot\left(\underline{\boldsymbol{K}}^{(k)} \cdot \nabla^{X} p^{f(k)}\right) \mathrm{d} V-\int_{B} \psi \frac{1}{M^{(k)} \Delta t}\left(p_{n+1}^{f(k)}-p_{n}^{f}\right) \mathrm{d} V
\end{aligned}
$$

with $J^{(k)}=J\left(\boldsymbol{x}^{(k)}\right)$ and $\underline{\boldsymbol{K}}^{(k)}=\underline{\boldsymbol{K}}\left(x^{(k)}\right)=\boldsymbol{J}^{(k)} \underline{\boldsymbol{F}}^{(k)-1} \cdot \underline{\boldsymbol{k}} \cdot \underline{\boldsymbol{F}}^{(k)-T}$. The discretization leads to

$$
\begin{aligned}
\tilde{\boldsymbol{\psi}}^{T} \underline{\boldsymbol{K}}_{p}^{(k)} \delta \tilde{\boldsymbol{p}}^{f} & =\tilde{\boldsymbol{\psi}}^{T} \int_{B}\left(\underline{\boldsymbol{h}}_{\chi}^{T} \underline{\boldsymbol{K}}^{(k)} \underline{\boldsymbol{h}}{ }_{\chi}+\frac{1}{\boldsymbol{M}^{(k)} \Delta t} \boldsymbol{h}^{T} \boldsymbol{h}\right) \mathrm{d} V \delta \tilde{\boldsymbol{p}}^{f}, \\
\tilde{\boldsymbol{\psi}}^{T} \boldsymbol{R}_{e x t}^{e} & =\tilde{\boldsymbol{\psi}}^{T} \int_{\partial B} \boldsymbol{h}^{T}\left(\boldsymbol{q}^{f} \cdot \boldsymbol{N}\right) \mathrm{d} A \\
\tilde{\boldsymbol{\psi}}^{T} \boldsymbol{R}_{J}^{e(k)} & =\tilde{\boldsymbol{\psi}}^{T} \int_{B} \boldsymbol{h}^{T}\left(B \frac{\ln J_{n+1}^{(k)}-\ln J_{n}}{\Delta t}\right) \mathrm{d} V \\
\tilde{\boldsymbol{\psi}}^{T} \boldsymbol{R}_{p}^{e(k)} & =\tilde{\boldsymbol{\psi}}^{T} \int_{B} \underline{\boldsymbol{h}}_{\chi}^{T} \underline{\boldsymbol{K}}^{(k)} \underline{\boldsymbol{h}}_{\chi} \mathrm{d} V \tilde{\boldsymbol{p}}^{f(k)} \\
& +\tilde{\boldsymbol{\psi}}^{T} \int_{B} \frac{1}{M^{(k)} \Delta t} \boldsymbol{h}^{T} \boldsymbol{h} \mathrm{d} V\left(\tilde{\boldsymbol{p}}^{f(k)}-\tilde{\boldsymbol{p}}_{n}^{f}\right) .
\end{aligned}
$$




\section{Verification of transient coupled solid-fluid interaction}

In order to verify the poromechanical ALE formulation by comparison to a transient computation, the transient poromechanical computation has to be verified first. This is performed in the following by comparing it to an analytical solution, which is, for example, available for the Terzaghi problem. The discretized system and the prescribed boundary conditions are shown in Figure A.1. The system is discretized by 30 isoparametric 20-node elements of different heights (finer at the top, coarser at the bottom). Starting with an initially unloaded system (displacements and pore pressure are zero), the soil is loaded by a constant load of $\sigma_{3}=-90 \mathrm{kN} / \mathrm{m}^{2}$ for $t>0$ at the top. The total loading time $t_{\text {end }}$, which is given in Table A.1 and differs for all three cases because of different permeabilities, is divided into 250 constant time steps. Three different fully saturated elastic soil materials (modeled by a hyperelastic neo-Hookean model; Table A.1) and the different sequential iteration schemes are examined. The application of the drained split leads to oversized displacements in the solid step and nonconverging behavior for the sequential solid-fluid iteration. The drained iteration scheme is, therefore, subsequently no longer considered. For comparison of the iteration schemes, the tolerance for the residual is prescribed to an absolute value (in cases 1 and $2\|\boldsymbol{r}\| \leqslant 0.1 \mathrm{kN}$ and in case $3\|\boldsymbol{r}\| \leqslant$ $10 \mathrm{kN}$ ) to obtain comparable numbers of required iteration steps. The resulting pore pressure and vertical displacements over the height for case 1 are illustrated in Figure A.2 for several times. The comparison of the pore pressure to the analytical solution (cf. [1]) shows good agreement. It can be seen that the fluid carries initially a big part of the load, except close to the surface, where the structure

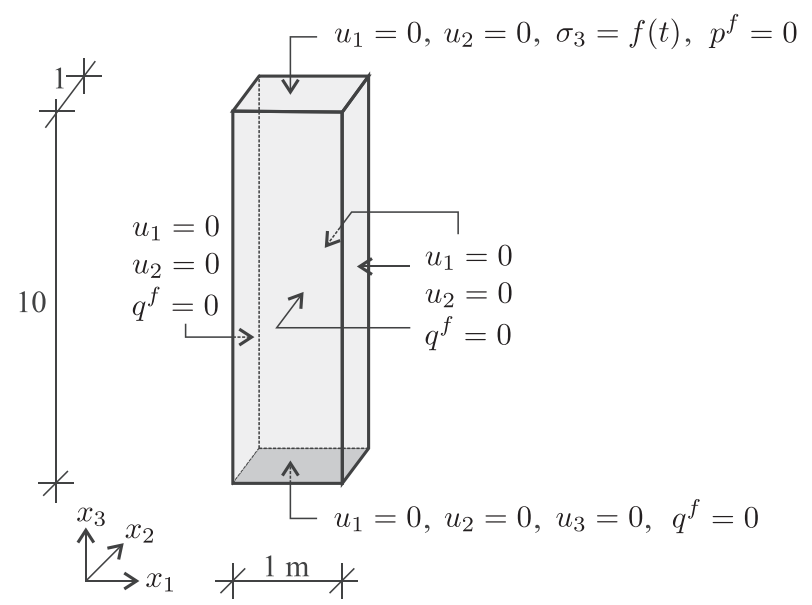

Figure A.1. System for the transient computation of Terzaghi problem for verification.

Table A.1. Material parameters for verification with Terzaghi example.

\begin{tabular}{lcccc}
\hline Solid & $\begin{array}{c}\mu \\
{\left[\mathrm{kN} / \mathrm{m}^{2}\right]}\end{array}$ & $\begin{array}{c}K \\
{\left[\mathrm{kN} / \mathrm{m}^{2}\right]}\end{array}$ & $\begin{array}{c}K_{s} \\
{\left[\mathrm{kN} / \mathrm{m}^{2}\right]}\end{array}$ & $\begin{array}{c}B \\
{[-]}\end{array}$ \\
\hline Case 1 & 22500 & 40000 & $4 \cdot 10^{8}$ & 1 \\
Case 2 & 4000 & 100000 & $4 \cdot 10^{8}$ & 1 \\
Case 3 & 4000 & 4000 & $4 \cdot 10^{8}$ & 1 \\
\hline Fluid & $k$ & $\hat{\phi}^{f}$ & $K_{f}$ & $t_{\text {end }}$ \\
& {$\left[\mathrm{m}^{4} / \mathrm{kNs}\right]$} & {$[-]$} & {$\left[\mathrm{kN} / \mathrm{m}^{2}\right]$} & {$[s]$} \\
\hline Case 1 & $3.06 \cdot 10^{-6}$ & 0.3 & 80000 & 500 \\
Case 2 & $5.0 \cdot 10^{-2}$ & 0.3 & 28600 & 0.05 \\
Case 3 & $1.0 \cdot 10^{-4}$ & 0.3 & 2200000 & 125 \\
\hline
\end{tabular}



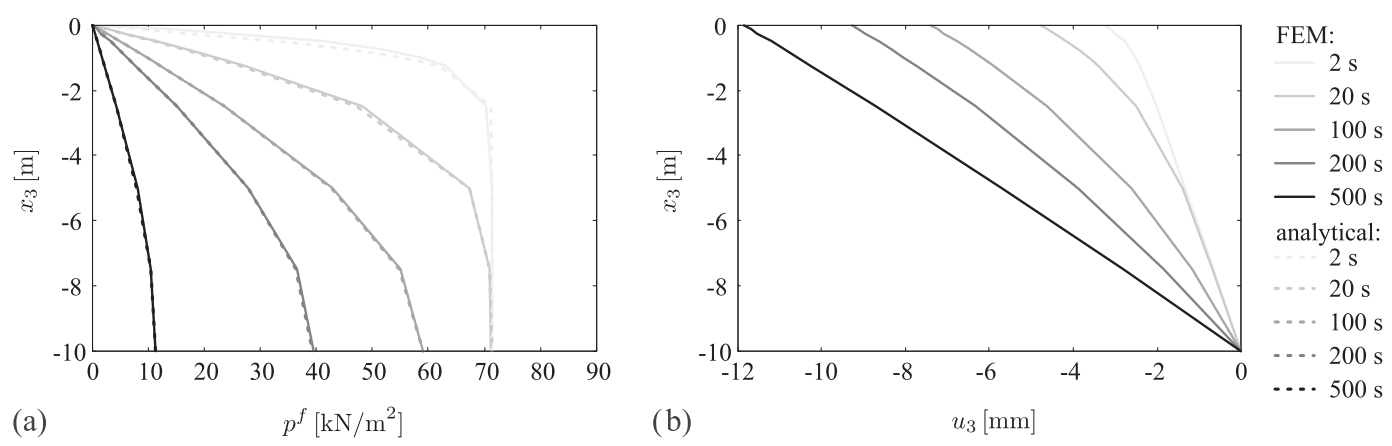

Figure A.2. Results of Terzaghi example case 1: (a) pore pressure over height compared with analytical solution and (b) vertical displacements over height
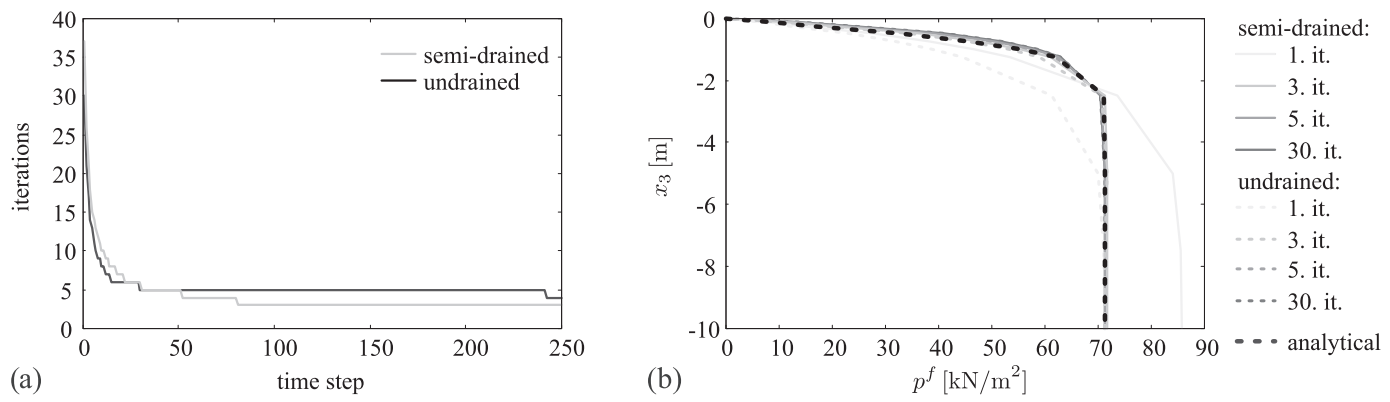

Figure A.3. Iterations of Terzaghi example case 1: (a) required iteration steps over time steps of semi-drained and undrained computation and (b) pore pressure over height in sequential iteration of first time step.

is drained. There, the solid has to carry the load from the beginning. This leads to the nonlinear distribution of the vertical displacements over the height. Over time, the excess pore pressure is dissipated through diffusion over the whole height, so that the pore pressure finally becomes zero, and the solid carries whole load once the porous medium reaches the drained limit. This leads to the final linear distribution of vertical displacements over height.

The required number of staggered iteration steps at the semi-drained and the undrained scheme is given in Figure A.3(a). They show comparable values. At the beginning, a larger quantity of iterations is required to meet the sharp gradient of the pore pressure distribution close to the drainage surface. Over time, the number of required iteration steps decreases. It is further interesting to have a closer look to the evolution of the pore pressure distribution over the single iteration steps, which is given in Figure A.3(b) for both schemes at the first time step. Because the undrained scheme is stiffer compared with the semi-drained scheme in the solid step, the undrained pore pressure values are smaller than the semi-drained ones in the first iteration step because of the smaller displacements. Further, it can be seen that the pore pressure distribution at both schemes is already at the third iteration step close to the analytical solution and, after the fifth step, the changes are no more visible. Therefore, it can be stated that the tolerance criterion is quite strict and could be relaxed to decrease the computational effort. Figure A.4 shows the required iteration steps versus the time steps as well as the pore pressure distribution over the height compared with the analytical solution at several times for case 2 .

Again, both iteration schemes show comparable results, and the pore pressure distribution fits the analytical results. This situation changes in case 3 , where the number of required iteration steps is quite high especially for the first time steps, where the iteration stops at the prescribed maximum number of iterations (900) in the undrained scheme. In case 3, the semi-drained iteration scheme requires less staggered steps than the undrained scheme (Figure A.5(a)). The evolution of the pore pressure distribution over the height during iteration at the first step is given in Figure A.5(b). It can 

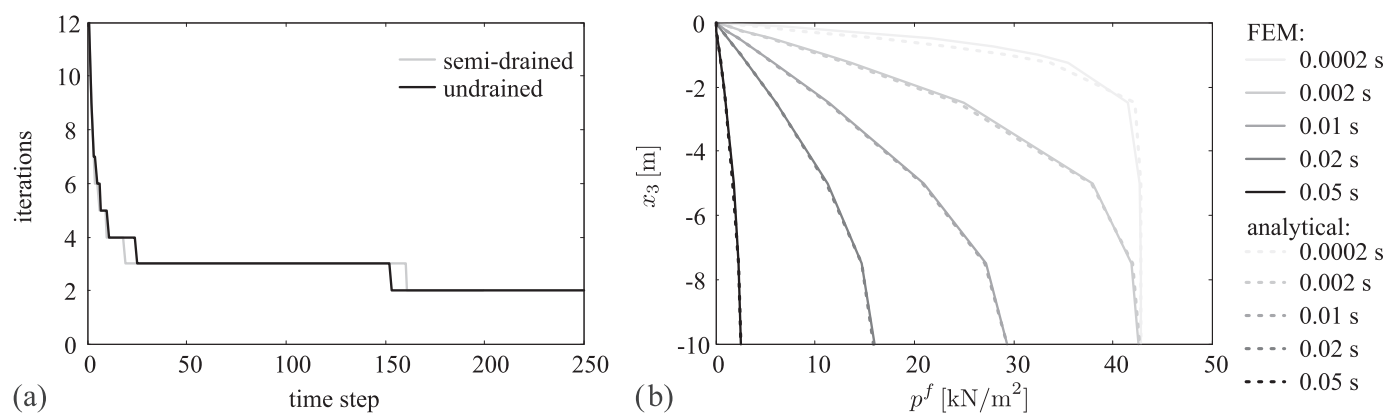

Figure A.4. Results of Terzaghi example case 2: (a) required iteration steps over time steps of semi-drained and undrained computation and (b) pore pressure over height compared with analytical solution.

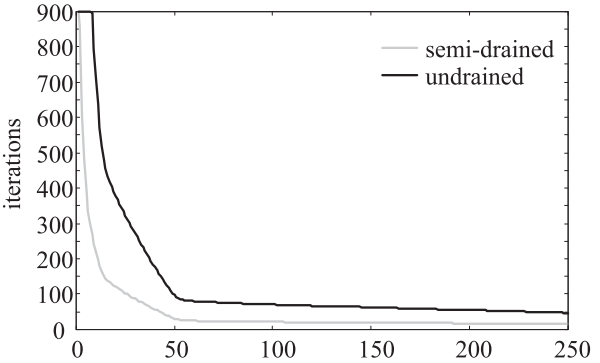

(a)

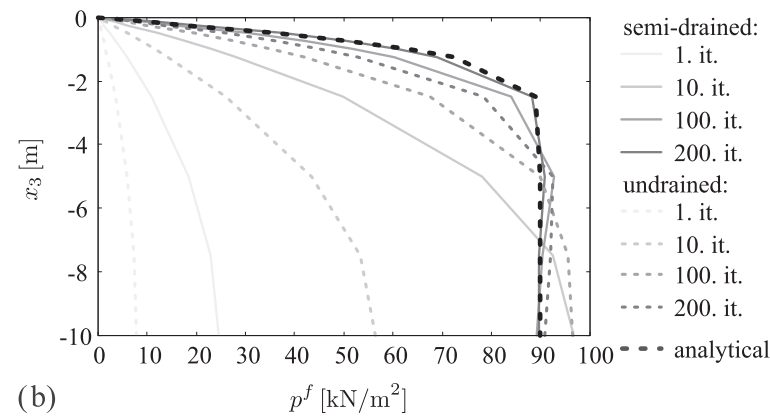

Figure A.5. Iterations of Terzaghi example case 3: (a) required iteration steps over time steps of semi-drained and undrained computation and (b) pore pressure over height in sequential iteration of first time step.
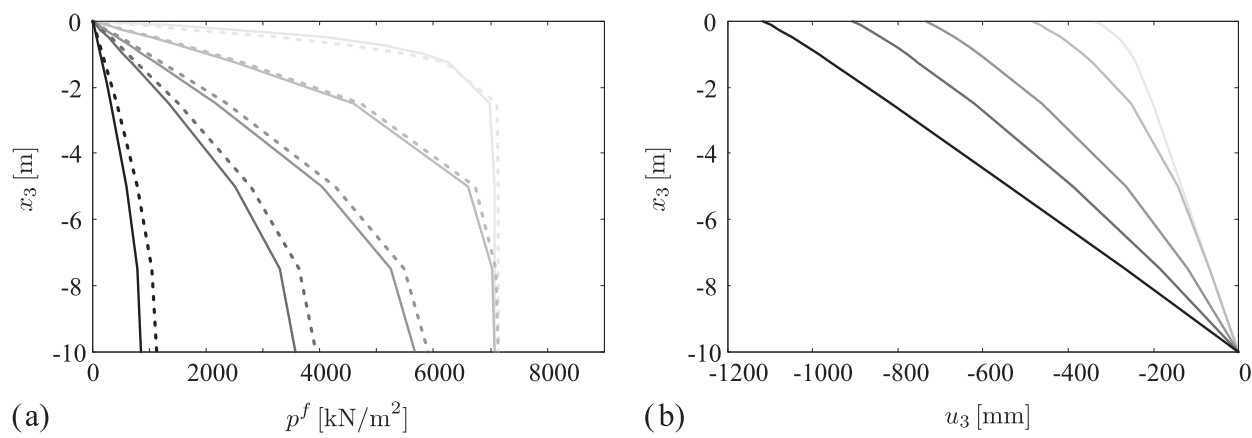

FEM:

$2 \mathrm{~s}$

$-20 \mathrm{~s}$

$-100 \mathrm{~s}$

$-200 \mathrm{~s}$

$-500 \mathrm{~s}$

analytical:

$2 \mathrm{~s}$

-..2 $20 \mathrm{~s}$

-... $100 \mathrm{~s}$

…200s

(b)

… $500 \mathrm{~s}$

Figure A.6. Results of Terzaghi example case 1 at large strain: (a) pore pressure over height compared with analytical solution and (b) vertical displacement over height.

be seen that the pore pressure in the first step is much too low in both cases, whereby the semi-drained one converges much faster, because of the softer behavior in the solid step. Nevertheless, also in case 3, the undrained and semi-drained scheme converges, and the analytical results are reached for all time steps (not illustrated). By the presented comparison to the analytical solution, the transient staggered solid-fluid iteration scheme of the poromechanical formulation is verified successfully.

As the last step of the verification, the transient scheme is tested at large strain. Therefore, the load of the Terzaghi example is increased by factor 100 to $\sigma_{3}=-9000 \mathrm{kN} / \mathrm{m}^{2}$. The results for material parameter case 1 are illustrated in Figure A.6. Because the analytical solution is restricted to small strains, the discrepancy between FEM results and the analytical solution increases with increasing displacements versus time. At $500 \mathrm{~s}$, the displacements at the top reach a value of $-1110 \mathrm{~mm}$ at a 

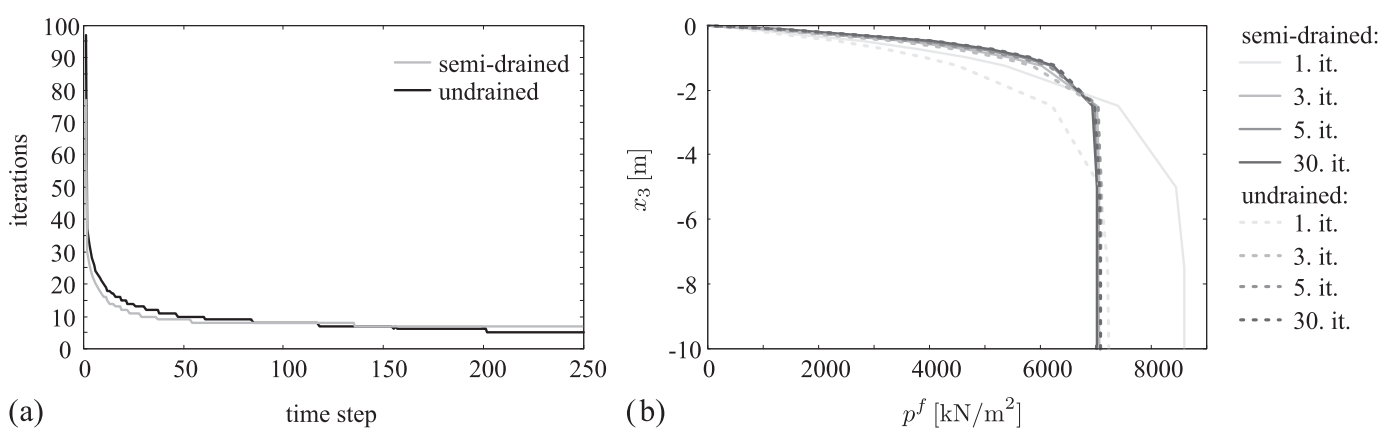

Figure A.7. Iterations of Terzaghi example case 1 at large strain: (a) required iteration steps over time steps of semi-drained and undrained computation and (b) pore pressure over height in sequential iteration of first time step.

total height of $10 \mathrm{~m}$, which corresponds to more than $10 \%$ of vertical strain. Because the tolerance criterion is not changed compared with the computation with $\sigma_{3}=-90 \mathrm{kN} / \mathrm{m}^{2}$, the number of required iteration steps increases expectably (Figure A.7(a). Although, regarding the evolution of the pore pressure distribution at the staggered iteration in the first time step in Figure A.7(b), it can be seen that the fifth step already shows no more visible discrepancies compared with the final solution.

\section{Verification of the poromechanical Arbitrary Lagrangian-Eulerian formulation}

In order to verify the sequential solid-fluid ALE solution scheme, a comparison of the ALE results to the transient total Lagrangian computation is carried out. Therefore, an example with equivalent boundary conditions in the systems of the ALE as well as transient computation is designed. The undrained sequential solid-fluid solver is applied for this verification. The system for the transient example is a cube with a side length of $1 \mathrm{~m}$ (Figure A.8), which is discretized by 10 20-node isoparametric finite elements in the direction of $x_{3}$. The displacements $u_{1}$ and $u_{2}$ are fixed for all nodes, and the displacements $u_{3}$ are fixed at the bottom. A time-dependent displacement $u_{3}=f(t)$ is defined as loading at the top. For the fluid part, the pore pressure is prescribed to zero at the bottom, while all other boundaries are undrained $q^{f}=0$. Thus, the fluid mass flux occurs only in $x_{3}$-direction. Initially, at $t=0 \mathrm{~s}$, the pore pressure is zero at the whole cube. A comparable ALE computation is performed with the system illustrated in Figure A.9. The cuboid is $10 \mathrm{~m}$ long, $1 \mathrm{~m}$ high, and $1 \mathrm{~m}$ wide and discretized by 50 elements in the $\chi_{1}$-direction and again 10 elements in the $\chi_{3}$-direction. The displacements $\hat{\mathrm{u}}_{1}$ and $\hat{\mathrm{u}}_{2}$ are fixed at the whole cuboid, and at the bottom of the cuboid, $\hat{\mathrm{u}}_{3}$ is fixed additionally. The material flows at the velocity $w_{1}$ from the left-hand to the right-hand side through this body. Thus, the boundary on the left side is the boundary where the material flows into the system. At this boundary, the initial conditions at $t=0 \mathrm{~s}$ of the transient computation are fulfilled in the ALE computation by prescribing the initial pore pressure $p^{f}=0$ and the initial vertical displacement at the top $\hat{\mathrm{u}}_{3}\left(\chi_{1}=0 \mathrm{~m}\right)=u_{3}(t=0 \mathrm{~s})$. Because of the guiding velocity $w_{1}$, a material particle reaches the position $\chi_{1}$ at the time $t\left(\chi_{1}\right)=\chi_{1} / w_{1}$. Therefore, the displacements at the top are prescribed to $\hat{u}_{3}\left(\chi_{1}\right)=f\left(\chi_{1}\right)=f\left(t=\chi_{1} / w_{1}\right)=u_{3}\left(t=\chi_{1} / w_{1}\right)$. The pore pressure at the bottom is prescribed to zero, and all other boundaries without prescribed pore pressure are undrained. In order to ensure that the mass flux occurs analogously to the transient computation only in the $\chi_{3}$ direction, the permeabilities in the $\chi_{1}$-direction and $\chi_{2}$-direction are chosen much smaller than in the $\chi_{3}$-direction. The mechanical properties of the solid are modeled by a hyperelastic neo-Hookean model. The material parameters of case 1 given in Table A.1 are utilized. Then, the results in the cross section (with constant $\chi_{1}$ ) can be compared with the results of the transient computation at time $t=\chi_{1} / w_{1}$. The comparison is performed for the two different load functions shown in Figure A.10, different guiding velocities, and varying displacement amplitudes $\hat{u}_{e}$.

First, the transient and the ALE computation are performed with load function $f_{1}$, the maximum displacement at the top of $\hat{\mathrm{u}}_{e}=-1 \mathrm{~cm}$, and a guiding velocity $w_{1}=1 \mathrm{~m} / \mathrm{s}$. In this case, the material 


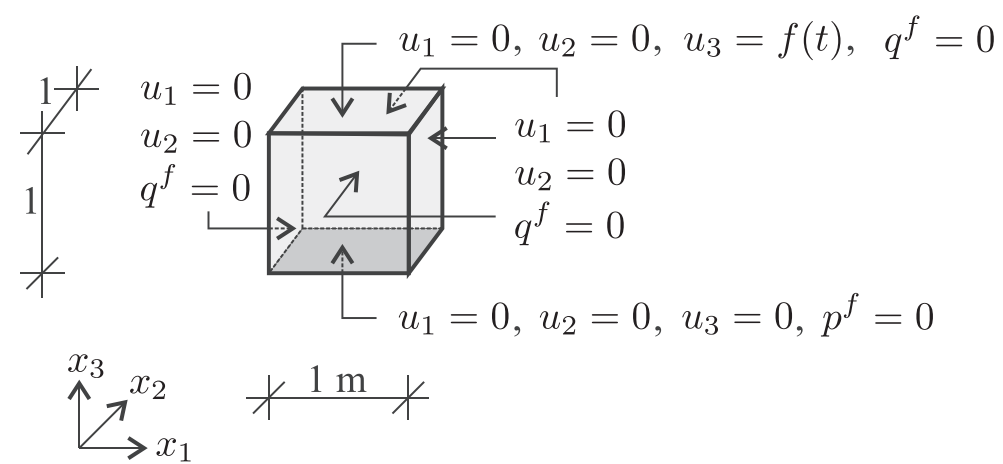

Figure A.8. System of the transient computation for verification.

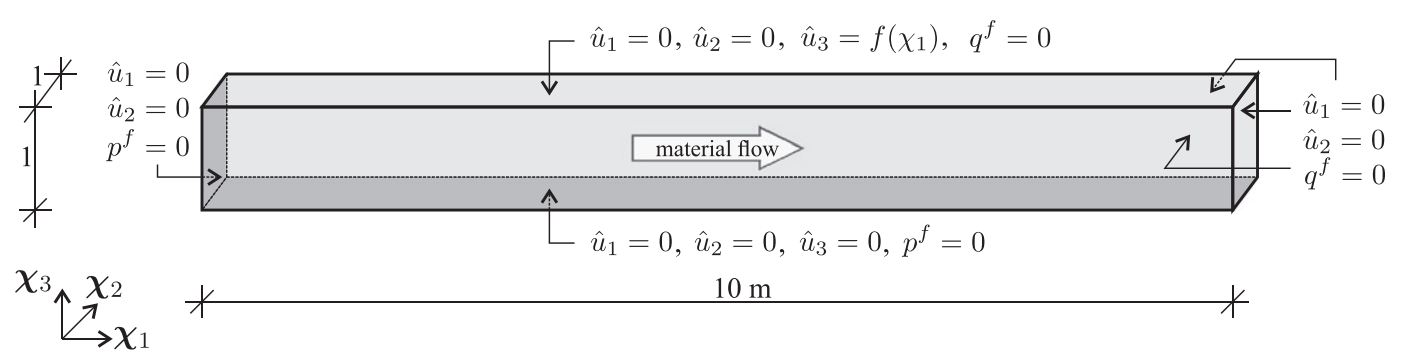

Figure A.9. System of the arbitrary Lagrangian-Eulerian computation for verification.

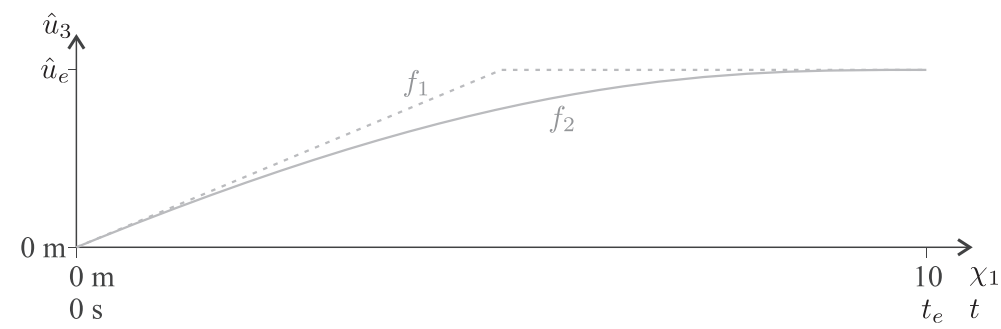

Figure A.10. Load functions of the verification example.

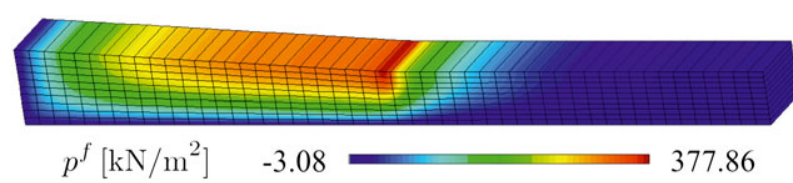

Figure A.11. Pore pressure of the arbitrary Lagrangian-Eulerian computation for load function $f_{1}, \hat{\mathrm{u}}_{e}=$ $-1 \mathrm{~cm}$ and $w_{1}=1 \mathrm{~m} / \mathrm{s}$ (scaled displacements).

takes $10 \mathrm{~s}$ to flow through the ALE system, which is therefore the total time of the transient computation. A pre-study shows that a number of 500 time steps with constant time step length of $0.02 \mathrm{~s}$ leads to converged results in the transient computation. The ALE computation is performed in one step. The corresponding pore pressure distribution is illustrated in Figure A.11. It can be seen that the pore pressure increases at the top because of the increasing displacement. The maximum pore pressure is reached at the top close to the kink in the middle of the system. On the right-hand side of the kink, the pore pressure decreases because the displacements are kept constant, and the fluid 


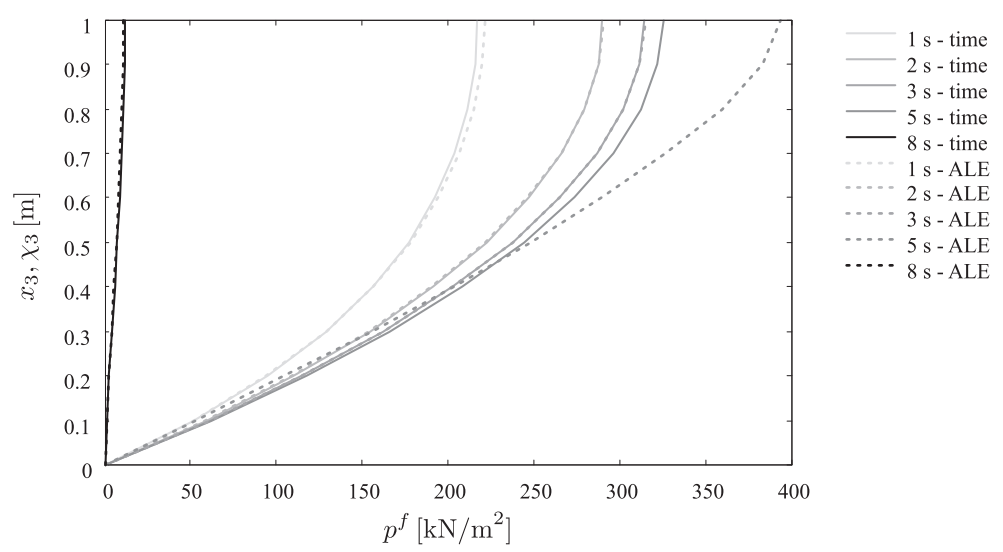

Figure A.12. Comparison of transient and arbitrary Lagrangian-Eulerian (ALE) results for load function $f_{1}$, $\hat{\mathrm{u}}_{e}=-1 \mathrm{~cm}$ and $w_{1}=1 \mathrm{~m} / \mathrm{s}$

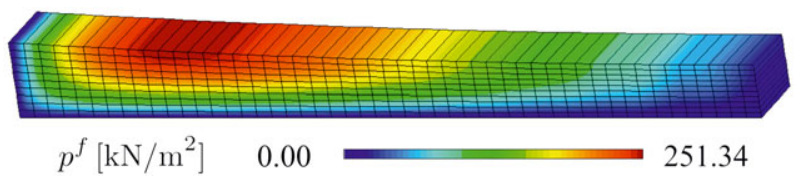

Figure A.13. Pore pressure at arbitrary Lagrangian-Eulerian computation for load function $f_{2}, \hat{\mathrm{u}}_{e}=-1 \mathrm{~cm}$ and $w_{1}=1 \mathrm{~m} / \mathrm{s}$ (scaled displacements).
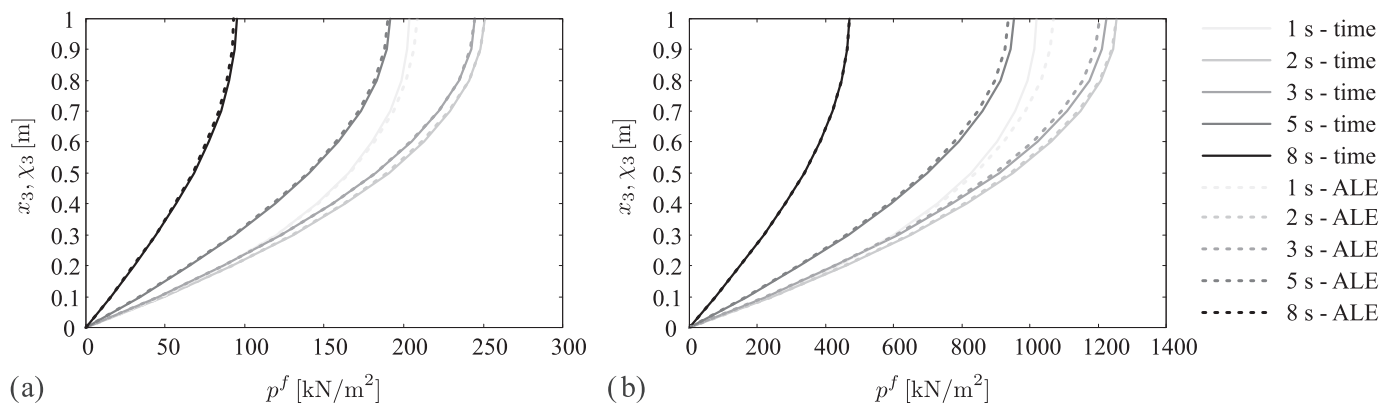

Figure A.14. Comparison of transient and arbitrary Lagrangian-Eulerian (ALE) results for load function $f_{2}$, $w_{1}=1 \mathrm{~m} / \mathrm{s}$ and (a) $\hat{\mathrm{u}}_{e}=-1 \mathrm{~cm}$ and (b) $\hat{\mathrm{u}}_{e}=-5 \mathrm{~cm}$.

is drained in vertical direction. Figure A.12 shows the comparison of the ALE results and the transient computation, whereby the values of the ALE results at certain time $t_{r}$ are taken from the cross section with $\chi_{1 r}=t_{r} \cdot w_{1}$. Almost all results of the transient and the ALE computation coincide quite well. Only at $t=5 \mathrm{~s}$, which is exactly at the kink, the ALE results show a local error.

Second, the transient and the ALE computations are carried out with a smooth load function $f_{2}$, two different maximum displacements at the top of $\hat{\mathrm{u}}_{e}=-1 \mathrm{~cm}$ and $\hat{\mathrm{u}}_{e}=-5 \mathrm{~cm}$, and two different guiding velocities of $w_{1}=1 \mathrm{~m} / \mathrm{s}$ as well as $w_{1}=5 \mathrm{~m} / \mathrm{s}$. In case of $w_{1}=1 \mathrm{~m} / \mathrm{s}$, the material takes again $10 \mathrm{~s}$ to flow through the ALE system, while at $w_{1}=5 \mathrm{~m} / \mathrm{s}$, the total time is $2 \mathrm{~s}$. Again, 500 time steps with a constant time step length of 0.02 and $0.004 \mathrm{~s}$, respectively, are used for the transient computation. The resulting pore pressure distribution for $\hat{\mathrm{u}}_{e}=-1 \mathrm{~cm}$ and $w_{1}=1 \mathrm{~m} / \mathrm{s}$ is illustrated in Figure A.13. Now, a smooth distribution of the pore pressure can be observed, which is first increasing (from the left to the right) at the top due to the fast increasing displacement until a maximum value is reached at around $\chi_{1}=2 \mathrm{~m}$. Afterwards, the drainage and the corresponding 

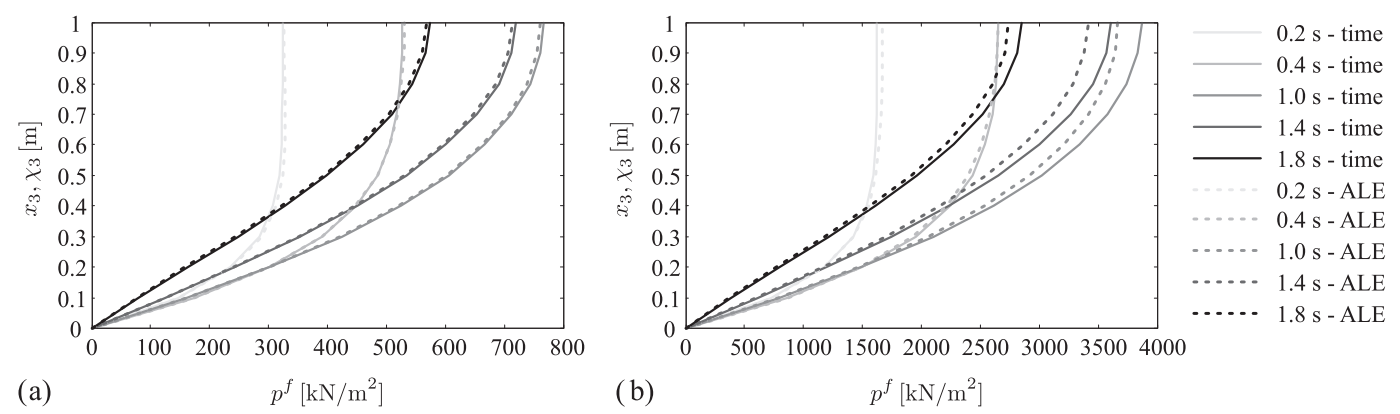

Figure A.15. Comparison of transient and arbitrary Lagrangian-Eulerian (ALE) results for load function $f_{2}$, $w_{1}=5 \mathrm{~m} / \mathrm{s}$ and (a) $\hat{\mathrm{u}}_{e}=-1 \mathrm{~cm}$ and (b) $\hat{\mathrm{u}}_{e}=-5 \mathrm{~cm}$.

decrease of the pore pressure predominate the increase of pore pressure because of the slower increasing displacements. In Figures A.14 and A.15, the results of the ALE and the transient computations for the different displacements and guiding velocities are depicted. The good agreement of the results verifies successfully the poromechanical ALE formulation.

\section{ACKNOWLEDGMENTS}

This research is partially supported by the Excellence Initiative of German Federal and State Governments (Institutional Strategy, measure 'Support the Best'), Deutsche Forschungsgemeinschaft (DFG) under Grant KA 1163/31, the Technische Universität Dresden through the Dresden Junior Fellowship, the Earth Materials and Surface Processes program at the US Army Research Office under grant contract W911NF-14-1-0658 and W911NF-15-1-0581, the US Air Force Office of Scientific Research Young Investigator Program Award, as well as the Mechanics of Material and Structure program at National Science Foundation under grant contract CMMI-1462760. These supports are gratefully acknowledged.

\section{REFERENCES}

1. von Terzaghi K, Rendulic L. Die wirksame Flächenporosität des Betons. Zeitschrift des Österreichischen Ingenieurund Architekten-Vereins 1934; 86:1-9.

2. Biot M. General theory of three dimensional consolidation. Journal of Applied Physics 1941; 12:155-164.

3. Borja RI, Tamagnini C, Alarcón E. Elastoplastic consolidation at finite strain part 2: finite element implementation and numerical examples. Computer Methods in Applied Mechanics and Engineering 1998; 159:103-122.

4. Armero F. Formulation and finite element implementation of a multiplicative model of coupled poro-plasticity at finite strains under fully saturated conditions. Computer Methods in Applied Mechanics and Engineering 1999; 171:205-241.

5. Sanavia L, Schrefler B, Steinmann P. A formulation for an unsaturated porous medium undergoing large inelastic strains. Computational Mechanics 2002; 28:137-151.

6. Sun W, Ostien JT, Salinger AG. A stabilized assumed deformation gradient finite element formulation for strongly coupled poromechanical simulations at finite strain. International Journal for Numerical and Analytical Methods in Geomechanics 2013; 37:2755-2788.

7. Sun W. A stabilized finite element formulation for monolithic thermo-hydro-mechanical simulations at finite strain. International Journal for Numerical Methods in Engineering 2015; 103:798-839.

8. Nackenhorst U. The ALE-formulation of bodies in rolling contact theoretical foundations and finite element approach. Computer Methods in Applied Mechanics and Engineering 2004; 193:4299-4322.

9. Donea J. Arbitrary Lagrangian-Eulerian finite element models. In Computational Methods for Transient Analysis, Belytschko T, Hughes T (eds). Elsevier: Amsterdam; 473-516, 1983.

10. Hughes T, Liu W, Zimmermann T. Lagrangian-Eulerian finite element formulation for incompressible viscous flows. Computer Methods in Applied Mechanics and Engineering 1981; 29:329-349.

11. Wang J, Gadala M. Formulation and survey of ALE method in nonlinear solid mechanics. Finite Elements in Analysis and Design 1997; 24:253-269.

12. Rodriguez-Ferran A, Casadei F, Huerta A. ALE stress update for transient and quasistatic processes. International Journal for Numerical Methods in Engineering 1998; 43:241-262. 
13. Tadi Beni Y, Movahhedy M. Consistent arbitrary Lagrangian Eulerian formulation for large deformation thermomechanical analysis. Materials and Design 2012; 31:3690-3702.

14. Wollny I, Hartung F, Kaliske M. Numerical modeling of inelastic structures at loading of steady state rolling: thermomechanical asphalt pavement computation. Computational Mechanics 2016; 57:867-886.

15. Nasdala L, Kaliske M, Becker A, Rothert H. An efficient viscoelastic formulation for steady state rolling. Computational Mechanics 1998; 22:395-403.

16. Ziefle M, Nackenhorst U. Numerical techniques for rolling rubber wheels: treatment of inelastic material properties and frictional contact. Computational Mechanics 2008; 42:337-356.

17. Behnke R, Wollny I, Kaliske M. Thermo-mechanical analysis of steady state rolling tires by numerical simulation and experiment. In Constitutive Models for Rubber VIII, Gil-Negrete Laborda N, Alonso A (eds). CRC Press, Taylor \& Francis Group: London, 2013:123-130.

18. Suwannachit A, Nackenhorst U. A novel approach for thermomechanical analysis of stationary rolling tires within an ALE-kinematic framework. Tire Science and Technology 2013; 41:174-195.

19. Behnke R, Kaliske M. Thermo-mechanically coupled investigation of steady state rolling tires by numerical simulation and experiment. International Journal of Non-Linear Mechanics 2015; 68:101-131.

20. Wollny I, Kaliske M. Numerical simulation of pavement structures with inelastic material behavior under rolling tyres based on an arbitrary Lagrangian Eulerian (ALE) formulation. Road Materials and Pavement Design 2013; 14:71-89.

21. Kaliske M, Wollny I, Behnke R, Zopf C. Holistic analysis of the coupled vehicle-tire-pavement system for the design of durable pavements. Tire Science and Technology 2015; 43:86-116.

22. Nazem M, Sheng D, Carter JP, Sloan SW. Arbitrary Lagrangian-Eulerian method for large-strain consolidation problems. International Journal for Numerical and Analytical Methods in Geomechanics 2008; 32:1023-1050.

23. Ishigami G, Miwa A, Nagatani K, Yoshida K. Terramechanics-based model for steering maneuver of planetary exploration rovers on loose soil. Journal of Field Robotics 2007; 24:233-250.

24. Melanz D, Jayakumar P, Negrut D. Experimental validation of a differential variational inequality-based approach for handling friction and contact in vehicle/granular-terrain interaction. Journal of Terramechanics 2016; 65:1-13.

25. Donea J, Huerta A, Ponthot JP, Rodriguez-Ferran A. Arbitrary Lagrangian-Eulerian methods. In Encyclopedia of Computational Mechanics (vol.1: Fundamentals), Stein E, de Borst R, Hughes T (eds). John Wiley \& Sons: Chichester; 414-437, 2004.

26. Bowen RM. Incompressible porous media models by use of the theory of mixtures. International Journal of Engineering Science 1980; 18:1129-1148.

27. Zienkiewicz OC, Chan A, Pastor M, Schrefler B, Shiomi T. Computational Geomechanics. John Wiley \& Sons: Chichester, 1999.

28. Wang K, Sun W. A semi-implicit discrete-continuum coupling method for porous media based on the effective stress principle at finite strain. Computer Methods in Applied Mechanics and Engineering 2016; 304:546-583.

29. Coussy O. Mechanics and Physics of Porous Solids. John Wiley \& Sons: Chichester, 2011.

30. Holzapfel GA. Nonlinear Solid Mechanics: A Continuum Approach for Engineering. John Wiley \& Sons: Chichester, 2000.

31. Nur A, Byerlee J. An exact effective stress law for elastic deformation of rock with fluids. Journal of Geophysical Research 1971; 76:6414-6419.

32. Ehlers W, Zinatbakhsh S, Markert B. Stability analysis of finite difference schemes revisited: a study of decoupled solution strategies for coupled multifield problems. International Journal for Numerical Methods in Engineering $2013 ; 94: 758-786$.

33. Jha B, Juanes R. A locally conservative finite element framework for the simulation of coupled flow and reservoir geomechanics. Acta Geotechnica 2007; 2:139-153.

34. Kim J, Tchelepi HA, Juanes R. Stability, accuracy and efficiency of sequential methods for coupled flow and geomechanics. SPE Journal 2011; 16(2):249-262.

35. Kim J, Tchelepi H, Juanes R. Stability and convergence of sequential methods for coupled flow and geomechanics: drained and undrained splits. Computer Methods in Applied Mechanics and Engineering 2011; 200:2094-2116.

36. Kim J, Tchelepi H, Juanes R. Stability and convergence of sequential methods for coupled flow and geomechanics: fixed-stress and fixed-strain splits. Computer Methods in Applied Mechanics and Engineering 2011; 200:1591-1606.

37. Castelletto N, White J, Tchelepi H. Accuracy and convergence properties of the fixed-stress iterative solution of twoway coupled poromechanics. International Journal for Numerical and Analytical Methods in Geomechanics 2015; 39:1593-1618.

38. White JA, Castelletto N, Tchelepi HA. Block-partitioned solvers for coupled poromechanics: a unified framework. Computer Methods in Applied Mechanics and Engineering 2016; 303:55-74.

39. Wollny I, Behnke R, Villaret K, Kaliske M. Numerical modelling of tyre-pavement-interaction phenomena: coupled structural investigations. Road Materials and Pavement Design 2016; 17:563-578. 